\title{
Different Anticoagulant Regimens, Mortality, and Bleeding in Hospitalized Patients with COVID-19: A Systematic Review and an Updated Meta-Analysis
}

\author{
Roberta Parisi, MSc ${ }^{1}$ Simona Costanzo, $\mathrm{PhD}^{1}$ Augusto Di Castelnuovo, $\mathrm{PhD}^{2}$ \\ Giovanni de Gaetano, MD, PhD ${ }^{1}$ Maria Benedetta Donati, MD, PhD ${ }^{1}$ Licia lacoviello, MD, PhD ${ }^{1,3}$ \\ ${ }^{1}$ Department of Epidemiology and Prevention. IRCCS Neuromed, via \\ dell'Elettronica, Pozzilli, Isernia, Italy \\ 2 Mediterranea Cardiocentro, Via Orazio n.2, Napoli, Italy \\ ${ }^{3}$ Research Center in Epidemiology and Preventive Medicine (EPIMED), \\ Department of Medicine and Surgery, University of Insubria, Varese,

\begin{abstract}
Address for correspondence Simona Costanzo, PhD, Department of Epidemiology and Prevention, IRCCS Neuromed, via dell'Elettronica, 86077 Pozzilli, Isernia, Italy (e-mail: simona.costanzo@neuromed.it; simona.costanzo@moli-sani.org).
\end{abstract} Italy

Semin Thromb Hemost 2021;47:372-391.

\begin{abstract}
We conducted a systematic review and a meta-analysis to assess the association of anticoagulants and their dosage with in-hospital all-cause mortality in COVID-19 patients. Articles were retrieved until January 8,2021 , by searching in seven electronic databases. The main outcome was all-cause mortality occurred during hospitalization. Data were combined using the general variance-based method on the effect estimate for each study. Separate meta-analyses according to type of COVID-19 patients (hospitalized or intensive care unit [ICU] patients), anticoagulants (mainly heparin), and regimens (therapeutic or prophylactic) were conducted. A total of 29 articles were selected, but 23 retrospective studies were eligible for quantitative meta-analyses. No clinical trial was retrieved. The majority of studies were of good quality; however, $34 \%$ did not distinguish heparin from other anticoagulants. Meta-analysis on 25,719 hospitalized COVID-19 patients showed that anticoagulant use was associated with $50 \%$ reduced in-hospital mortality risk (pooled risk ratio [RR]: 0.50, 95\% confidence interval $[\mathrm{Cl}]: 0.40-0.62 ; \mathrm{l}^{2}: 87 \%$ ). Both anticoagulant regimens (therapeutic and prophylactic) reduced in-hospital all-cause mortality, compared with no anticoagulation. Particularly in ICU patients, the anticoagulant therapeutic regimen was associated with a reduced in-hospital mortality risk (RR: $0.30,95 \% \mathrm{Cl}: 0.15-0.60 ; I^{2}$ : $58 \%$ )

Keywords

- COVID-19

- coagulation

- heparin

- bleeding

- mortality compared with the prophylactic one. However, the former was also associated with a higher risk of bleeding (RR: $2.53,95 \% \mathrm{Cl}: 1.60-4.00 ; I^{2}$ : $65 \%$ ). Anticoagulant use, mainly heparin, reduced all-cause mortality in COVID-19 patients during hospitalization. Due to the higher risk of bleeding at therapeutic doses, the use of prophylactic dosages of anticoagulant is probably to be preferred in noncritically ill COVID-19 patients.
\end{abstract}

published online April 13, 2021
Issue Theme Maintaining Hemostasis and Preventing Thrombosis in COVID-19 -Part II; Guest Editors: Emmanuel J. Favaloro, PhD, FFSc (RCPA) and Giuseppe Lippi, MD. (c) 2021. Thieme. All rights reserved. Thieme Medical Publishers, Inc., 333 Seventh Avenue, 18th Floor, New York, NY 10001, USA
DOI https://doi.org/ $10.1055 / \mathrm{s}-0041-1726034$. ISSN 0094-6176. 
Histopathological investigations of fatal cases of coronavirus disease 2019 (COVID-19) reported that the primary cause of death was respiratory failure with exudative diffuse alveolar damage and massive capillary congestion. ${ }^{1,2}$ In addition, in these subjects, the frequent presence of extensive pulmonary interstitial fibrosis and pulmonary microthrombosis has been shown. These findings might explain the development of hypoxemia and respiratory failure, and support the concept of a hypercoagulable state in these critically ill patients. ${ }^{1,3}$

The severe acute respiratory syndrome coronavirus-2 (SARS-CoV-2) appears to generate a prothrombotic condition as evidenced by different reports of arterial, venous, and pulmonary-related thrombosis in COVID-19 patients. Indeed, a high incidence of thrombotic events and bleeding complications has been reported in patients with COVID$19 .^{4-7} \mathrm{~A}$ common finding in these patients requiring hospitalization is increased levels of D-dimer (i.e., a fibrin degradation product) and a longer prothrombin time, which are both associated with a higher risk of death. ${ }^{8}$

Heparin is able to bind SARS-CoV-2 spike protein and could act as a competitive inhibitor for viral entry, thus decreasing virus infectivity. ${ }^{9}, 10$ In addition, heparin has antiinflammatory effects, both at the vasculature and the airway levels, which could beneficially impact COVID-19-associated inflammation. ${ }^{10}$ Thus, anticoagulant treatment could improve the prognosis of COVID-19 patients. Despite the versatile role of heparin as both an anticoagulant and an antiinflammatory drug, and theoretical antiviral effect, no data from randomized clinical trials are available yet to prove the efficacy of this drug in COVID-19 patients.

Nevertheless, during the first months of pandemic outbreak, guidelines on thromboprophylaxis and anticoagulant therapy in COVID-19 were rapidly emerging, with different recommendations, ${ }^{9-15}$ focusing mainly on prevention of venous thromboembolism (VTE) events in COVID-19 patients.

The World Health Organization, the U.S. Centers for Disease Control and Prevention, and Department of Defense recommended a prophylactic dose of unfractionated heparin (UFH) or low-molecular-weight heparin (LMWH) for prevention of VTE in hospitalized adults and adolescents with severe COVID-19 disease, except if contraindicated. ${ }^{11-13}$

The Italian Society on Thrombosis and Haemostasis ${ }^{14}$ and a position paper endorsed by several international societies suggested VTE risk stratification for all individuals with COVID-19 and extended thromboprophylaxis postdischarge for patients at a higher risk of VTE, while recognizing insufficient evidence to recommend the empiric use of therapeutic doses of UFH and LMWH. ${ }^{6,15}$ Others have suggested intermediate or therapeutic doses of LMWH for hospitalized patients and extended VTE prophylaxis for up to 45 days postdischarge. ${ }^{15}$ Finally, the article by Barnes et al recommended pharmacologic VTE prophylaxis for all hospitalized nonpregnant patients with confirmed or highly suspected COVID-19, regardless of VTE risk assessment score, unless a contraindication exists; for patients who were being discharged from hospital, extended VTE prophylaxis was not suggested. ${ }^{16}$
Several randomized controlled clinical trials are currently ongoing, ${ }^{17,18}$ and preliminary data have recently been published from observational studies on the use of heparins or other anticoagulant drugs with contrasting results.

We therefore conducted a systematic review and performed a meta-analysis of published studies on the effects of anticoagulant use (i.e., heparin and nonheparin anticoagulants together) on in-hospital all-cause mortality, trying also to separate prophylactic from therapeutic anticoagulant dosage, to provide clinical insights for consideration in the management of hospitalized COVID-19 patients.

\section{Methods}

This study was conducted according to the recommendations outlined in the Cochrane Handbook for Systematic Reviews of Interventions. ${ }^{19}$ The protocol was registered at https://www.crd.york.ac.uk/prospero/ as CRD42020212915. Institutional review board approval was not required, as the study did not directly involve human participants.

\section{Search strategy}

A flow diagram for study selection is reported in -Supplementary Fig. S1. Articles published in Medline, Embase, PubMed, Web of Science, Cochrane Central Database, MedRxiv, and Preprints.org were retrieved until January 8,2021 . Studies were restricted to humans, and their titles and/or abstracts contained at least one of the following terms: "coronavirus," "COVID-19," or "SARS-CoV-2," plus the term "heparin," "anticoagulant treatment," or "low molecular weight," or "oral anticoagulant," or "direct thrombin inhibitors," plus the term "mortality," "death," or "survival." An assessment of references was also conducted. Additionally, we searched peer-reviewed international congress abstracts in the dedicated section on COVID-19.

We identified 330 publications. To be included in this systematic review, the study had to (1) include only COVID19 patients and (2) report qualitative and/or quantitative findings on the association of heparin (mentioned as such) or an anticoagulant treatment (including heparin or not) with mortality in COVID-19 patients.

Two of us (S.C. and R.P.) independently reviewed the identified studies, then jointly excluded the articles not adhering with one or both criteria and agreed on a final selection of 29 studies, ${ }^{20-48}$ including three published as preprints in MedRxiv, the preprint server for health science, ${ }^{21,24,45}$ and one congress abstract. ${ }^{27}$ No randomized controlled clinical trial was retrieved.

\section{Assessment of Methodological Quality}

Two investigators (S.C. and R.P.) independently assessed the methodological quality of each study by using the Newcastle-Ottawa Scale (NOS), ${ }^{49}$ developed to assess quality of nonrandomized studies such as cohort and case-control studies. The NOS rating for each study was then converted to the Agency for Healthcare Research and Quality standard. ${ }^{50}$ Disagreements were resolved by consensus or by a third investigator (A.D.C.), if consensus could not be reached. 


\section{Meta-Analysis: Data Extraction and Data Analysis}

The main meta-analysis was performed considering all studies that reported adjusted estimates of the effects of anticoagulant treatment on in-hospital all-cause mortality compared with no anticoagulant use in hospitalized COVID-19 patients. When both prophylactic and therapeutic dosages were compared with a referent group formed by nontreated patients, we included in the meta-analysis the effect estimate of the prophylactic dose. ${ }^{35,36,39}$ We performed different meta-analyses according to the characteristics of COVID-19 patients (all patients hospitalized or treated in the intensive care unit [ICU]) and to different types of anticoagulant dosage (therapeutic or prophylactic regimens). We also performed a subgroup meta-analysis considering only the studies that reported the association of specified heparin (i.e., LMWH and UFH) treatment with inhospital all-cause mortality.

A secondary meta-analysis was performed considering as outcome the bleeding events, the most representative adverse effect of anticoagulant use. In this case, the numbers of events in both anticoagulant and control groups were extracted and used to calculate risk ratio (RR) and 95\% confidence intervals (CIs) for each selected study.

All analyses were performed using standard statistical procedures provided in RevMan5.4 (the Cochrane Collaboration, Oxford, United Kingdom). Data were combined using the general variance-based method that requires information on the effect estimates and their $95 \% \mathrm{CI}$ from each study. In addition, 95\% CIs were used to assess the variance and the relative weight of each study. Heterogeneity was assessed using the Higgins' $I^{2}$ metric. When the heterogeneity among studies appeared to be high $\left(I^{2}>60 \%\right)$, results from the random effects model only were considered. The hypothesis that publication bias might have affected the validity of the estimates was visually tested by a funnel plot-based approach (-Supplementary Fig. S2).

\section{Results}

\section{Characteristics of the Studies}

The general characteristics of the 29 studies are shown in -Tables 1 and 2.

Four studies were from China, ${ }^{20,21,42,47} 14$ from Europe, ${ }^{22,23,28,29,31,32,34,35,40,41,43,44,46,48}$ and 11 from United States ( - Table 1). ${ }^{24-27,30,33,36-39,45}$ All were retrospective observational studies. Studies included ICU or hospitalized COVID-19 patients, except for the study by Tremblay et al that included both ambulatory and hospitalized COVID-19 patients. ${ }^{25}$ All studies included male and female adults. The sample size ranged from 26 to 4,389 patients (-Table $\mathbf{1}$ ). In general, the studies collected retrospective data (i.e., treatment, outcome, comorbidity, COVID-19 severity) from patient electronic medical records and defined mortality as death occurred during hospitalization for any cause ("overall" or "all-cause"). In particular, the majority of the studies $(N=25)$ considered in-hospital all-cause mortality as the primary outcome (-Table $\mathbf{2}$ ), while the remaining focused mainly on thrombotic or bleeding complications ${ }^{21,22,28}$ or on acute respiratory distress syndrome. $^{29}$

Eighteen studies reported data exclusively for heparin (UFH or LMWH) treatment. ${ }^{20-23,28,30-32,34,35,40-44,46-48}$ Six studies investigated the role of any anticoagulant treatment, including LMWH or UFH, direct thrombin inhibitors, and/or direct oral anticoagulants. ${ }^{24,33,36-38,45}$ Only one study investigated three types of anticoagulant drugs separately (i.e., apixaban, enoxaparin, UFH). ${ }^{39}$ No information was provided on the type of anticoagulant used by the remaining four studies (- Table 2). ${ }^{25-27,29}$ The studies mainly used as a reference a group formed by patients not treated with any anticoagulant. $^{20,21,23,25-27,29,31-37,39,41,42,45,47,48}$ Additionally, 13 studies compared two groups of patients at different dosages of anticoagulant (therapeutic vs. prophylactic) (-Table 2). ${ }^{22,24,28,30,33,34,37,38,40,43,44,46,48}$ The studies were mostly considered of good quality (23/29) (-Supplementary Table S1). ${ }^{50}$ Wide heterogeneity was found regarding the outcomes investigated (domain 3 ), the type of anticoagulant used, and the definition of the dosage (domains 1 and 2). In particular, each study had its own definition of therapeutic or prophylactic dosage, without a standard dosage of reference.

\section{Qualitative Review: Association with Mortality}

\section{Anticoagulant Use versus No Anticoagulant Use}

Studies comparing patients who received anticoagulants or not $^{20,21,23,25-27,29,31-37,39,41,42,45,47,48}$ differed from each other in type and dosage of treatment, and showed conflicting results (-Table $\mathbf{2}$ ). The study of Tang et al was the first that investigated the association between anticoagulant treatment and 28-day mortality in 449 Chinese COVID-19 patients ( $22 \%$ treated with therapeutic doses of LMWH); it reported that anticoagulant therapy was associated with a better prognosis only in severe COVID-19 patients with a higher risk of sepsis-induced coagulopathy or with markedly elevated D-dimer levels. ${ }^{20}$

Among the studies considering all hospitalized COVID-19 patients $(N=15)$, the majority reported that anticoagulant treatment was associated with lower in-hospital all-cause mortality (-Table 3). ${ }^{23,26,31-37,39,41,42,45,47,48}$ In particular, three studies conducted in large settings of hospitalized COVID-19 patients showed that anticoagulant treatment, either at therapeutic or prophylactic doses, was associated with a reduced risk of in-hospital mortality, compared with no anticoagulant treatment (- Table 3). ${ }^{36,37,48}$ Billett et al, investigating the efficacy of three types of anticoagulant drugs (i.e., apixaban, enoxaparin, UFH) on in-hospital mortality in COVID-19 hospitalized patients, observed that apixaban and enoxaparin had similar beneficial effects on that outcome. $^{39}$

On the contrary, the study of Tremblay et al concluded that anticoagulant therapy alone was unlikely to be protective for COVID-19-related morbidity and all-cause mortality. ${ }^{25} \mathrm{How}-$ ever, the latter study considered both outpatients and hospitalized COVID-19 patients and had a sample size relatively 
Anticoagulants, Mortality, and Bleeding in COVID-19 Patients Parisi et al. 375

Table 1 General characteristics of the 29 selected studies on anticoagulant treatment and risk of in-hospital mortality in COVID-19 patients

\begin{tabular}{|c|c|c|c|c|c|c|}
\hline Study & Country & Time period & $\begin{array}{l}\text { Type of } \\
\text { COVID-19 } \\
\text { patients }\end{array}$ & $N$ & $\begin{array}{l}\text { Sex, } \\
\text { male \% }\end{array}$ & $\begin{array}{l}\text { Age (y), } \\
\text { mean (SD) }\end{array}$ \\
\hline $\begin{array}{l}\text { Tang et al } 20 \\
\text { J Thromb Haemost } \\
\text { 2020, April } 27\end{array}$ & China & $\begin{array}{l}\text { From Jan. } 1 \text { to Feb. } 13, \\
2020\end{array}$ & $\begin{array}{l}\text { Severe COVID-19 } \\
\text { patients }\end{array}$ & 449 & 59.7 & $65.1(12.0)$ \\
\hline $\begin{array}{l}\text { Liu et } a^{21} \\
\text { Preprint from medRxiv } \\
2020 \text {, April } 28\end{array}$ & China & $\begin{array}{l}\text { From Feb. } 8 \text { to Mar. } 18 \text {, } \\
2020\end{array}$ & ICU patients & 61 & 67.2 & $72(10)$ \\
\hline $\begin{array}{l}\text { Llitjos et al } 22 \\
\text { J Thromb Haemost } \\
\text { 2020, May } 27\end{array}$ & France & $\begin{array}{l}\text { From Mar. } 19 \text { to Apr. 11, } \\
2020\end{array}$ & ICU patients & 26 & 77.0 & $\begin{array}{l}\text { Median } 68 \\
\text { IQR: } 51.5-74.5\end{array}$ \\
\hline $\begin{array}{l}\text { Ayerbe et al }{ }^{23} \\
\text { J Thromb Thrombolysis } \\
\text { 2020, May } 31\end{array}$ & Spain & $\begin{array}{l}\text { From Mar. } 1 \text { to Apr. 20, } \\
2020\end{array}$ & All patients & 2,075 & 60.5 & $67.6(15.5)$ \\
\hline $\begin{array}{l}\text { Trinh et } \mathrm{al}^{24} \\
\text { Preprint from medRxiv } \\
2020 \text {, June } 3\end{array}$ & United States & $\begin{array}{l}\text { From Mar. } 1 \text { to Apr. 11, } \\
2020\end{array}$ & ICU patients & 244 & 66.0 & $59.6(13.2)$ \\
\hline $\begin{array}{l}\text { Tremblay et al }{ }^{25} \\
\text { ASH } \\
2020 \text {, July } 2\end{array}$ & United States & $\begin{array}{l}\text { From Mar.1 to Apr. 1, } \\
2020\end{array}$ & $\begin{array}{l}\text { Ambulatory and } \\
\text { hospitalized } \\
\text { COVID-19 } \\
\text { patients }\end{array}$ & 656 & 44.7 & 69.1 (13.87) \\
\hline \multirow{2}{*}{$\begin{array}{l}\text { Paranjpe et } \mathrm{al}^{26} \\
\text { JACC } \\
2020 \text {, july } 7\end{array}$} & \multirow[t]{2}{*}{ United States } & \multirow{2}{*}{$\begin{array}{l}\text { From Mar. } 14 \text { to Apr. 11, } \\
2020\end{array}$} & All patients & 2,773 & \multirow[t]{2}{*}{ NR } & \multirow[t]{2}{*}{ NR } \\
\hline & & & ICU patients & 395 & & \\
\hline $\begin{array}{l}\text { Al-Samkari et al }{ }^{27} \\
\text { Res Pract Thromb Haemost } \\
2020 \text {, July } 15\end{array}$ & United States & $\begin{array}{l}\text { From Mar. } 4 \text { to Apr. } 11, \\
2020\end{array}$ & ICU patients & 2,809 & 64.5 & $\begin{array}{l}\text { Median } 61 \\
\text { IQR: } 53-71\end{array}$ \\
\hline $\begin{array}{l}\text { Pesavento et al } \\
\text { J Thromb Haemost } \\
\text { 2020, July } 21\end{array}$ & Italy & $\begin{array}{l}\text { From Feb. } 26 \text { to Apr. } 6 \text {, } \\
2020\end{array}$ & All patients & 324 & 55.9 & $\begin{array}{l}\text { Median } 71 \\
\text { IQR: } 59-82\end{array}$ \\
\hline $\begin{array}{l}\text { Russo et al } \\
\text { Pharmacological Research } \\
2020 \text {, September }\end{array}$ & Italy & From Feb. to Apr. 2020 & All patients & 192 & 59.9 & $67.7(15.2)$ \\
\hline $\begin{array}{l}\text { Ferguson et al }{ }^{30} \\
\text { J Clin Pharmacol } \\
\text { 2020, September }\end{array}$ & United States & $\begin{array}{l}\text { From Mar. } 15 \text { to May } 8 \text {, } \\
2020\end{array}$ & ICU patients & 141 & NR & NR \\
\hline $\begin{array}{l}\text { Schiavone et al }{ }^{31} \\
\text { Int J Cardiol } \\
\text { 2020, } 8 \text { September }\end{array}$ & Italy & $\begin{array}{l}\text { From Feb. } 23 \text { to April 1, } \\
2020\end{array}$ & All patients & 844 & 61.7 & $63.4(16.1)$ \\
\hline $\begin{array}{l}\text { Desai et al }{ }^{32} \\
\text { Int J of Cardiology } \\
2020,22 \text { September }\end{array}$ & Italy & $\begin{array}{l}\text { From Feb. } 21 \text { to April 14, } \\
2020\end{array}$ & All patients & 575 & 66.1 & 64.8 (14.6) \\
\hline $\begin{array}{l}\text { Hsu et } \mathrm{al}^{33} \\
\text { Thromb Res } \\
2020,23 \text { September }\end{array}$ & United States & $\begin{array}{l}\text { From Feb. } 27 \text { to Apr. } 24, \\
2020\end{array}$ & All patients & 468 & 54.9 & $\begin{array}{l}\text { Median } 65.1 \\
\text { IQR: } 52-75.5\end{array}$ \\
\hline $\begin{array}{l}\text { Gonzalez-Porras et al } \\
\text { Rev Med Virol } \\
\text { 2020, } 24 \text { September }\end{array}$ & Spain & $\begin{array}{l}\text { From Mar. } 1 \text { to April 7, } \\
2020\end{array}$ & All patients & 690 & 58.8 & $\begin{array}{l}\text { Median } 72.5 \\
\text { IQR: } 64-85\end{array}$ \\
\hline $\begin{array}{l}\text { Albani et al } \\
\text { EClinicalMedicine } \\
2020,5 \text { October }\end{array}$ & Italy & $\begin{array}{l}\text { From Feb. } 20 \text { to May 10, } \\
2020\end{array}$ & All patients & 1,403 & 65.5 & $\begin{array}{l}\text { Median 70.5 } \\
\text { IQR: 59.9-78.5 }\end{array}$ \\
\hline $\begin{array}{l}\text { lonescu et al }{ }^{36} \\
\text { Eur J Haematol } \\
2020,11 \text { October }\end{array}$ & United States & $\begin{array}{l}\text { From Mar. } 13 \text { to May 5, } \\
2020\end{array}$ & All patients & 3,480 & 48.5 & $64.5(17.0)$ \\
\hline $\begin{array}{l}\text { Nadkarni et } \text { al }^{37} \\
\text { JACC } \\
2020,20 \text { October }\end{array}$ & United States & $\begin{array}{l}\text { From Mar. } 1 \text { to Apr. } 30 \text {, } \\
2020\end{array}$ & All patients & 4,389 & 66 & $\begin{array}{l}\text { Median } 65 \\
\text { IQR: } 53-77\end{array}$ \\
\hline $\begin{array}{l}\text { Lynn et al }{ }^{38} \\
\text { Thromb Res } \\
\text { 2020, } 5 \text { November }\end{array}$ & United States & $\begin{array}{l}\text { From Mar. } 15 \text { to May 31, } \\
2020\end{array}$ & All patients & 402 & 53.7 & $>18$ \\
\hline
\end{tabular}


Table 1 (Continued)

\begin{tabular}{|c|c|c|c|c|c|c|}
\hline Study & Country & Time period & $\begin{array}{l}\text { Type of } \\
\text { COVID-19 } \\
\text { patients }\end{array}$ & $N$ & $\begin{array}{l}\text { Sex, } \\
\text { male \% }\end{array}$ & $\begin{array}{l}\text { Age (y), } \\
\text { mean (SD) }\end{array}$ \\
\hline $\begin{array}{l}\text { Billett et } \mathrm{al}^{39} \\
\text { Thromb Haemost } \\
\text { 2020, } 13 \text { November }\end{array}$ & United States & $\begin{array}{l}\text { From Mar. } 1 \text { to May } 30 \text {, } \\
2020\end{array}$ & All patients & 3,625 & 52.6 & $>18$ \\
\hline $\begin{array}{l}\text { Bolzetta et al }{ }^{40} \\
\text { Aging Clin Exp Res } \\
2020,16 \text { November }\end{array}$ & Italy & $\begin{array}{l}\text { From Mar. } 31 \text { to May } 1 \text {, } \\
2020\end{array}$ & All patients & 81 & 38.1 & $81.4(11.9)$ \\
\hline $\begin{array}{l}\text { Falcone et al }{ }^{41} \\
\text { Open Forum Infect Dis } \\
2020,19 \text { November }\end{array}$ & Italy & $\begin{array}{l}\text { From Mar. } 4 \text { to April 30, } \\
2020\end{array}$ & All patients & 315 & 76.2 & $\begin{array}{l}70 \\
\text { IQR: } 57-80\end{array}$ \\
\hline $\begin{array}{l}\text { Qin et al }{ }^{42} \\
\text { Thromb Res } \\
\text { 2020, } 23 \text { November }\end{array}$ & China & $\begin{array}{l}\text { From Jan } 10 \text { to Feb } 28, \\
2020\end{array}$ & All patients & 749 & 48 & $60(15)$ \\
\hline $\begin{array}{l}\text { Jonmarker et } \mathrm{al}^{43} \\
\text { Crit Care } \\
2020,23 \text { November }\end{array}$ & Sweden & From Mar. to April, 2020 & ICU patients & 152 & 82.2 & $\begin{array}{l}61 \\
\text { IQR: 52-69 }\end{array}$ \\
\hline $\begin{array}{l}\text { Canoglu and Saylan }{ }^{44} \\
\text { Ann Saudi Med } \\
\text { 2020, } 3 \text { December }\end{array}$ & Turkey & $\begin{array}{l}\text { From Mar. } 11 \text { to April } \\
30,2020\end{array}$ & $\begin{array}{l}\text { Severe COVID-19 } \\
\text { patients }\end{array}$ & 154 & 62.3 & $60(20.5)$ \\
\hline $\begin{array}{l}\text { Rentsch et al }{ }^{45} \\
\text { Preprint from medRxiv } \\
202011 \text { December }\end{array}$ & United States & $\begin{array}{l}\text { From Mar. } 1 \text { to July } \\
31,2020\end{array}$ & All patients & 4,297 & 93.4 & $\begin{array}{l}68 \\
\text { IQR: } 58-75\end{array}$ \\
\hline $\begin{array}{l}\text { Martinelli et al }{ }^{46} \\
\text { Intern Emerg Med } \\
2021,3 \text { January }\end{array}$ & Italy & $\begin{array}{l}\text { From Mar. } 9 \text { to April 7, } \\
2020\end{array}$ & All patients & 278 & 65.1 & $\begin{array}{l}59 \\
\text { IQR: 49-67 }\end{array}$ \\
\hline \multirow{2}{*}{$\begin{array}{l}\text { Shen et al }{ }^{47} \\
\text { Cardiovasc Drugs Ther } \\
20214 \text { January }\end{array}$} & \multirow[t]{2}{*}{ China } & \multirow{2}{*}{$\begin{array}{l}\text { From Jan. } 26 \text { to Mar. 26, } \\
2020\end{array}$} & All patients & 525 & \multirow[t]{2}{*}{49.3} & \multirow[t]{2}{*}{$64(19)$} \\
\hline & & & ICU patients & 89 & & \\
\hline \multirow{2}{*}{$\begin{array}{l}\text { Di Castelnuovo et al } \\
\text { Thromb Haemost } \\
\text { 2021, } 7 \text { January }\end{array}$} & \multirow[t]{2}{*}{ Italy } & \multirow{2}{*}{$\begin{array}{l}\text { From Feb. } 19 \text { to May 23, } \\
2020\end{array}$} & All patients & 2,574 & \multirow[t]{2}{*}{61.6} & \multirow[t]{2}{*}{$66.8(15.2)$} \\
\hline & & & ICU patients & 327 & & \\
\hline
\end{tabular}

Abbreviations: ICU, intensive care unit; IQR, interquartile range; NR, not reported.

small $(N=656)$. Finally, Russo et al observed that anticoagulant treatment prior to hospital admission did not affect the risk of death during hospitalization (RR: 1.15 , 95\% CI: $0.29-2.57) .^{29}$

Five studies investigated ICU COVID-19 patients. ${ }^{21,26,27,47,48}$ A study from a single center in the United States reported that the incidence of in-hospital mortality was $29.1 \%$ for those treated with anticoagulants as compared with $62.7 \%$ in patients who did not receive anticoagulant treatment. ${ }^{26}$ In particular, two recent studies found that in-hospital LMWH treatment was associated with a lower mortality in ICU COVID-19 patients (- Table 2). ${ }^{47,48}$ In contrast, Al-Samkari et al failed to show any difference in survival rate between treated and untreated groups, in a greater cohort of 2,809 subjects. ${ }^{27}$ Finally, the non-peerreviewed study by Liu et al based on a small sample size of only 61 COVID-19 patients showed that LMWH treatment led to severe thrombocytopenia with fatal outcome (25/61 had severe thrombocytopenia, of whom 96\% did not survive). ${ }^{21}$

\section{Therapeutic versus Prophylactic Dosage}

Thirteen studies compared two different dosages of anticoagulant treatment. $22,24,28,30,33,34,37,38,40,43,44,46,48$ The defi- nitions of therapeutic or prophylactic dose were different among studies (-Table 2 ). In the majority of the works reporting heparins' dosages (66\%), the prophylactic dosage included UFH $<5,000 \mathrm{IU}$; enoxaparin 20 to $40 \mathrm{mg}$ /daily or $1 \mathrm{mg} / \mathrm{kg} /$ daily; therapeutic dosage included 5,000 $>$ UFH $<15,000 \mathrm{IU}$; enoxaparin $>40 \mathrm{mg} /$ daily or $1 \mathrm{mg} / \mathrm{kg}$ twice or three times daily (- Table 2 ). ${ }^{24,30,34,37,38,44,46,48}$

The study by Pesavento et al reported that the rate for overall mortality was 12.2 (95\% CI: 8.1-17.8) per 100 persons/month in patients who received LMWH prophylactic doses and 20.1 (95\% CI: 11.0-33.8) per 100 persons/month in those treated with higher doses, defined as subtherapeutic. ${ }^{28}$ Di Castelnuovo et al showed that both prophylactic and therapeutic regimens were effective in reducing mortality, the prophylactic doses to a higher extent (HR: $1.54,95 \% \mathrm{CI}: 1.06-2.25){ }^{48}$

Similar results were observed by Hsu et al, showing that the group who received a therapeutic anticoagulant had a higher 30-day mortality compared with those receiving standard and high-intensity prophylaxis (40 vs. 15 vs. $6 \%$, respectively, $p<0.001){ }^{33}$ Finally, the study by Lynn et al reported that therapeutic anticoagulation did not provide inhospital mortality benefit over thromboprophylaxis, independent of comorbidities or disease severity. ${ }^{38}$ 


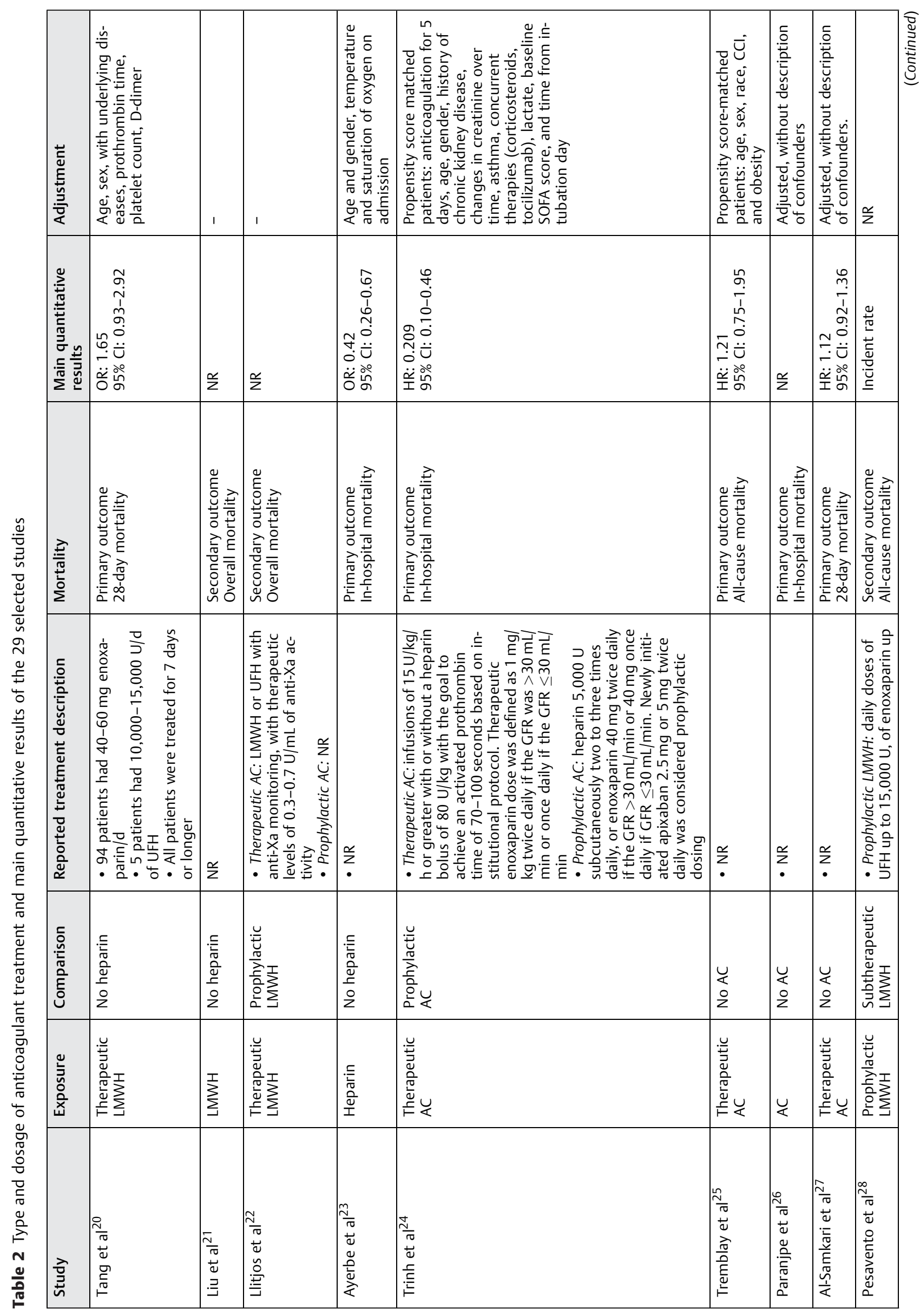




\begin{tabular}{|c|c|c|c|c|c|c|c|c|c|}
\hline \multirow{2}{*}{ 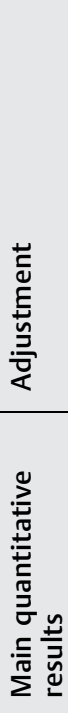 } & & \multirow{2}{*}{ 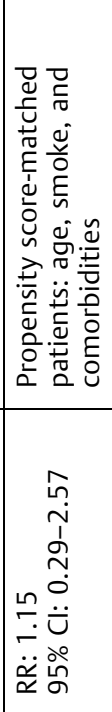 } & \multirow{2}{*}{ 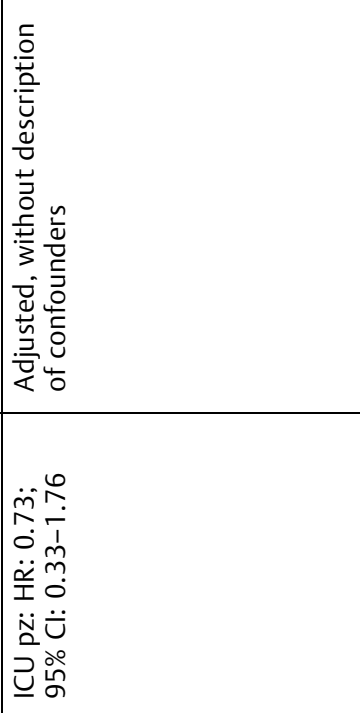 } & \multirow{2}{*}{\multicolumn{2}{|c|}{ 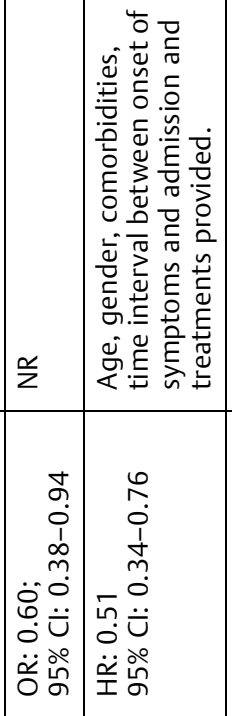 }} & \multicolumn{2}{|l|}{ 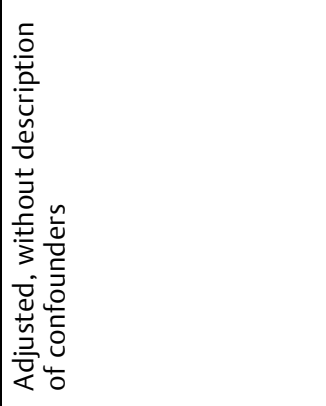 } & \multicolumn{2}{|l|}{ 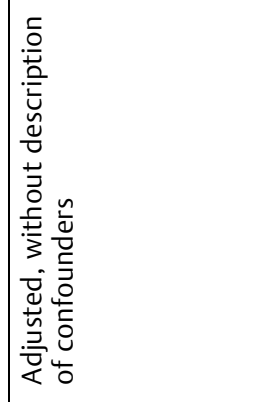 } \\
\hline & & & & & & 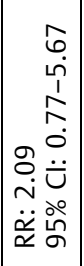 & 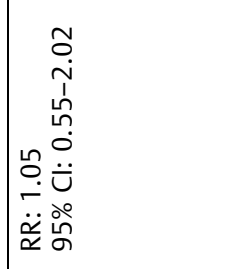 & 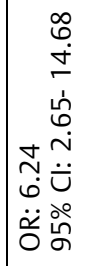 & 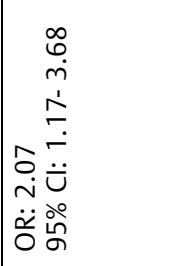 \\
\hline 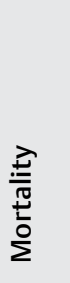 & & 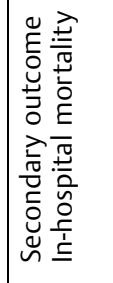 & 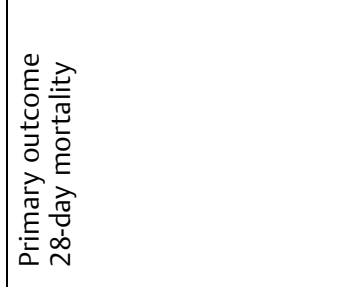 & 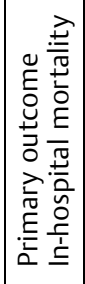 & 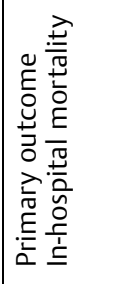 & \multicolumn{2}{|c|}{ 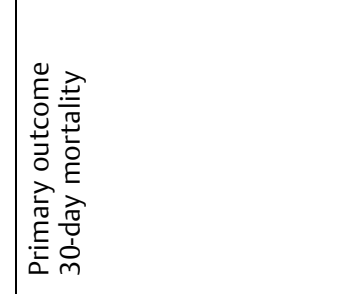 } & \multicolumn{2}{|c|}{ 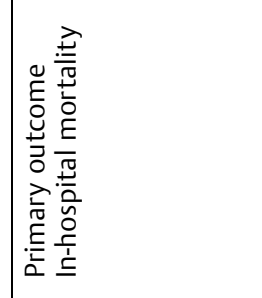 } \\
\hline 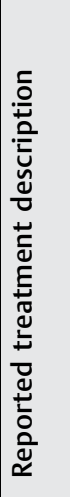 & 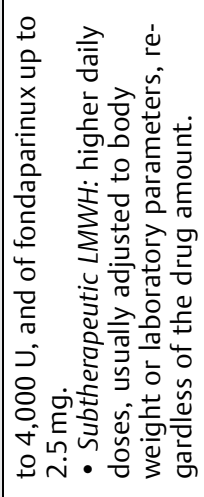 & 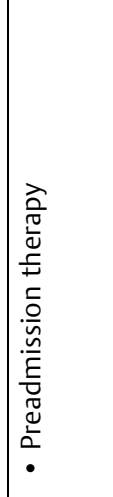 & 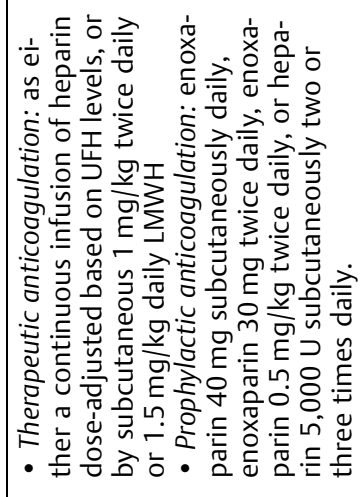 & $\begin{array}{l}\frac{\alpha}{z} \\
\end{array}$ & & 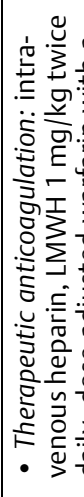 & 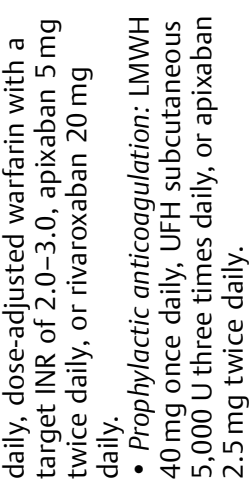 & 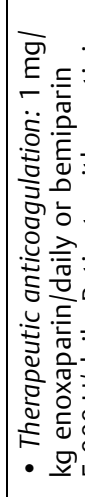 & 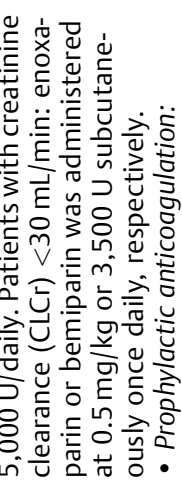 \\
\hline 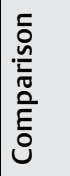 & & 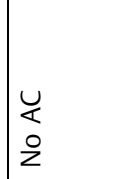 & 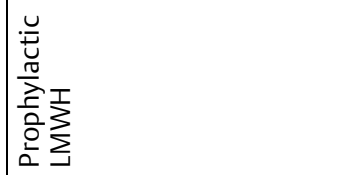 & 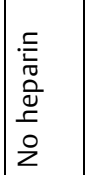 & 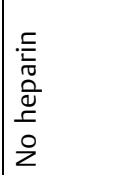 & 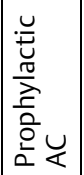 & 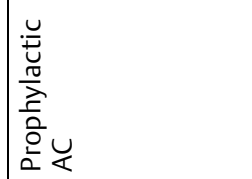 & 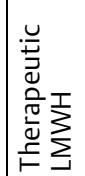 & 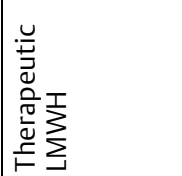 \\
\hline 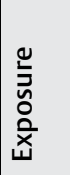 & & 茟 & 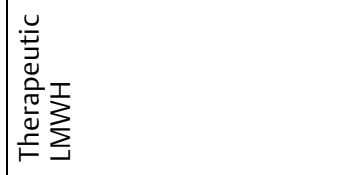 & 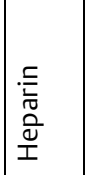 & 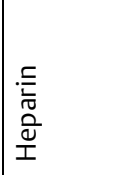 & 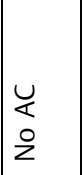 & 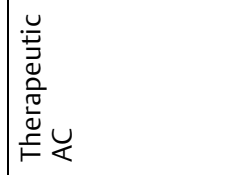 & $2 \stackrel{\sum}{\sum}$ & 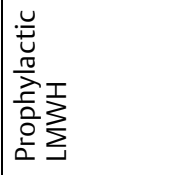 \\
\hline 剤 & & 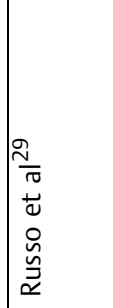 & 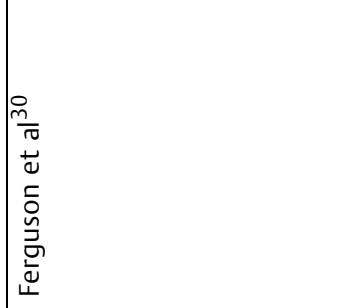 & 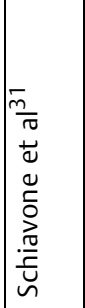 & 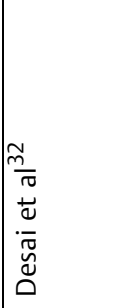 & 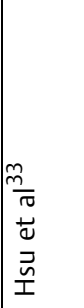 & & 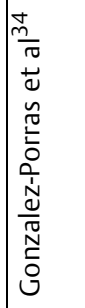 & \\
\hline
\end{tabular}




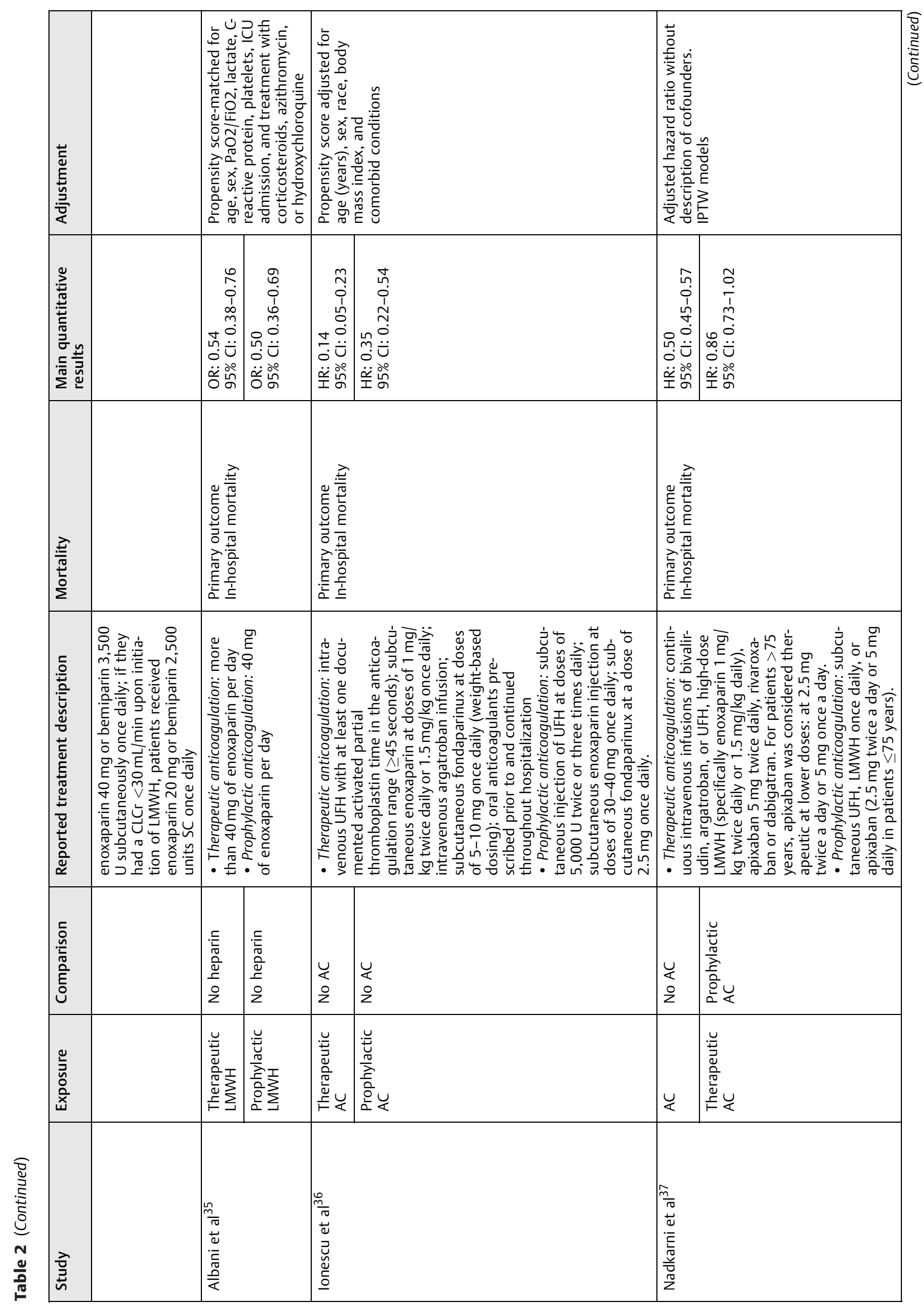




\begin{tabular}{|c|c|c|c|c|c|c|c|c|c|}
\hline \multirow{2}{*}{ 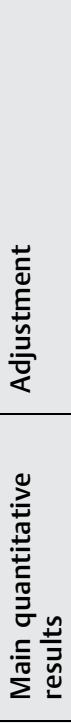 } & \multirow{2}{*}{ 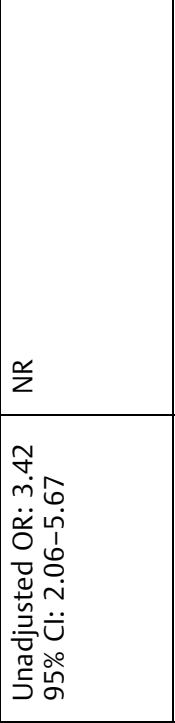 } & \multicolumn{2}{|c|}{ 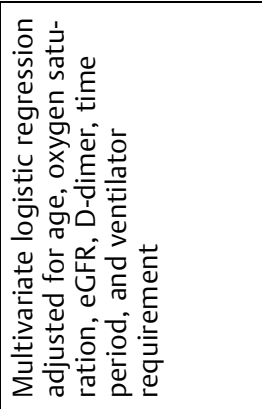 } & \multirow{2}{*}{ 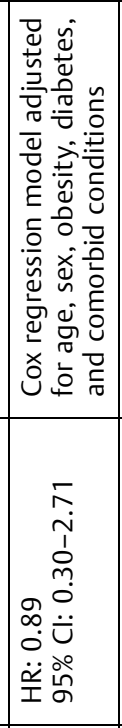 } & \multirow{2}{*}{ 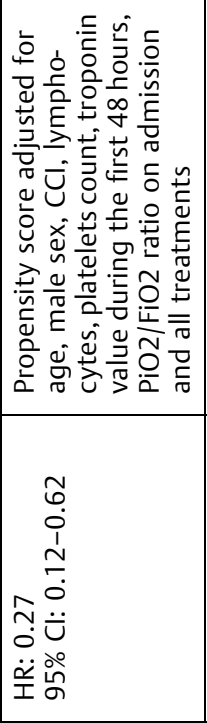 } & \multirow{2}{*}{ 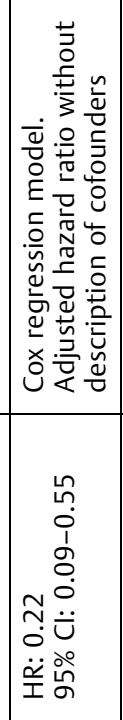 } & \multirow{2}{*}{ 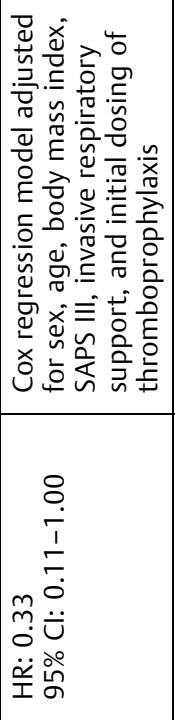 } & \multirow{2}{*}{ 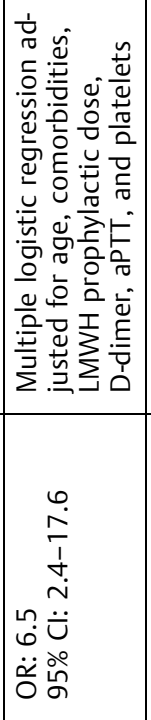 } & \multirow{2}{*}{ 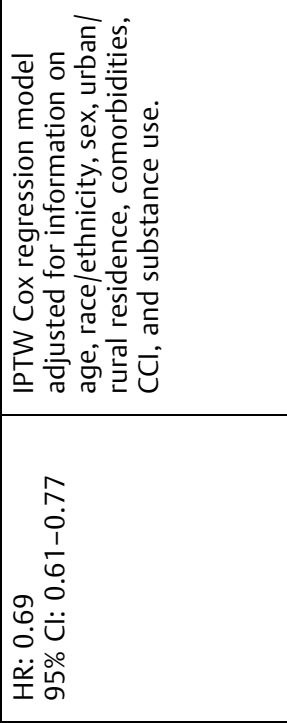 } \\
\hline & & 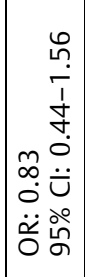 & 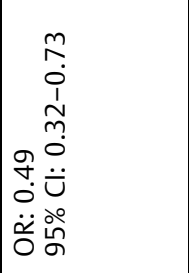 & & & & & & \\
\hline 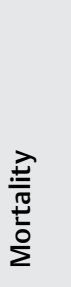 & 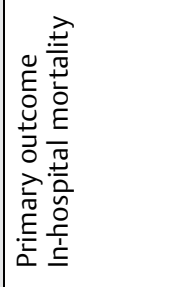 & \multicolumn{2}{|l|}{ 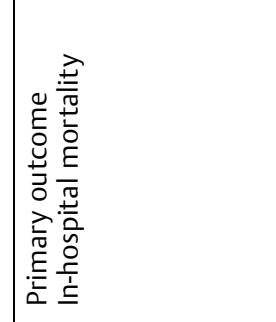 } & 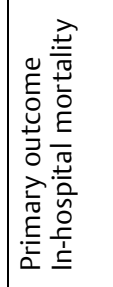 & 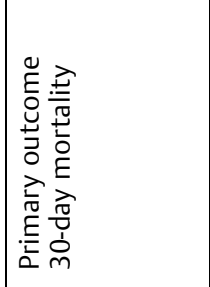 & 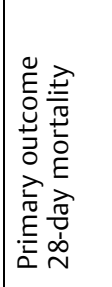 & 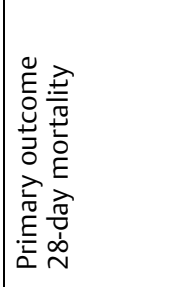 & 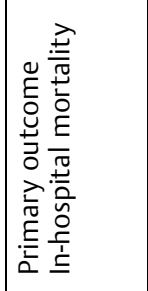 & 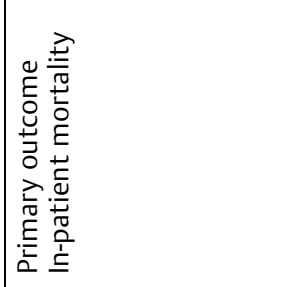 \\
\hline 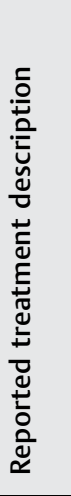 & 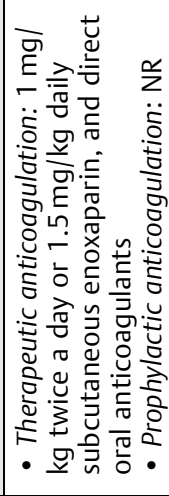 & 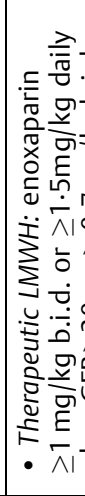 & 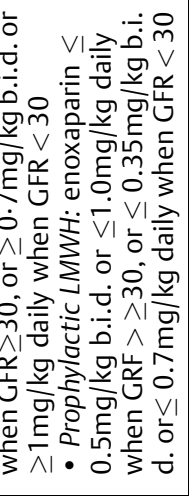 & 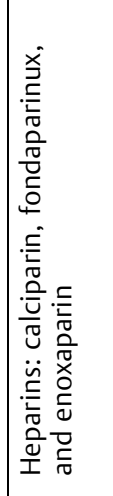 & 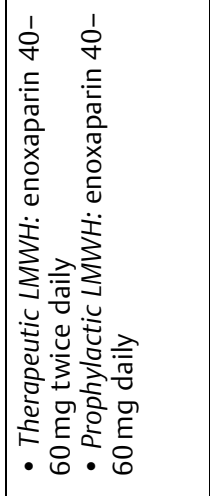 & 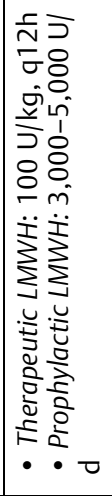 & 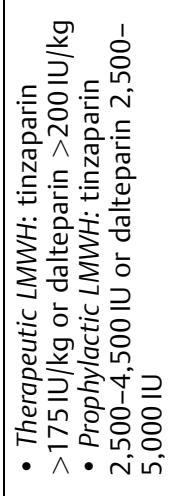 & 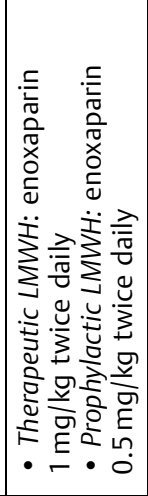 & 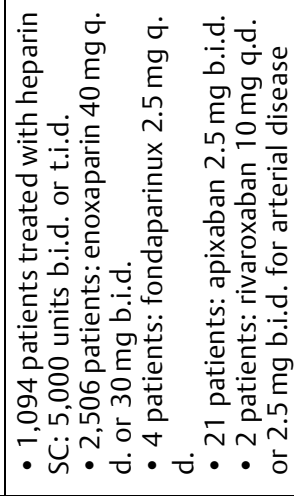 \\
\hline 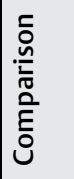 & 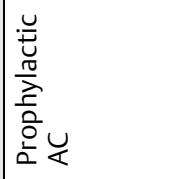 & 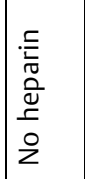 & 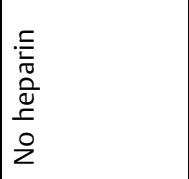 & 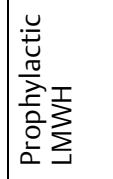 & 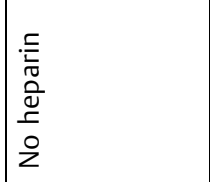 & 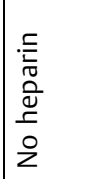 & 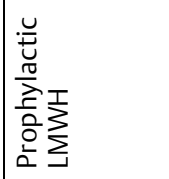 & 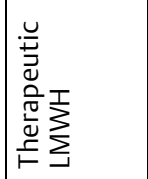 & 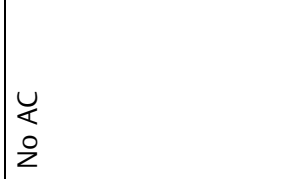 \\
\hline 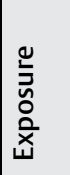 & 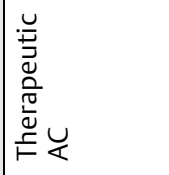 & 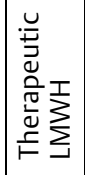 & 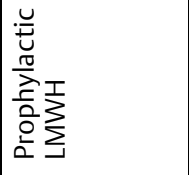 & 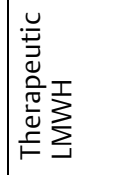 & $\sum_{\sum}^{I}$ & $\sum_{\sum}^{I}$ & 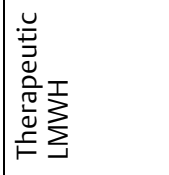 & 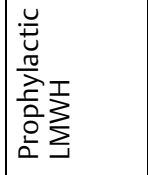 & $\ddot{\leftarrow}$ \\
\hline 离 & 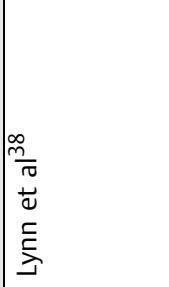 & 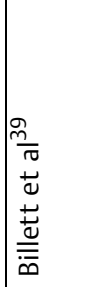 & & 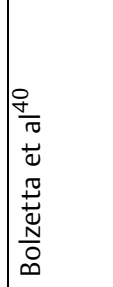 & 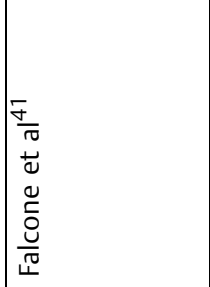 & $\begin{array}{l}\frac{\partial}{\pi} \\
\frac{\partial}{0} \\
\vdots \bar{\sigma}\end{array}$ & 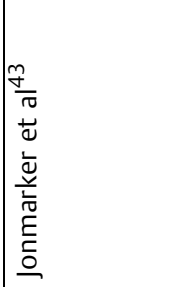 & 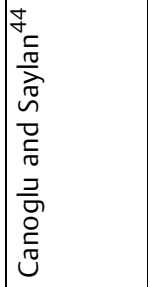 & 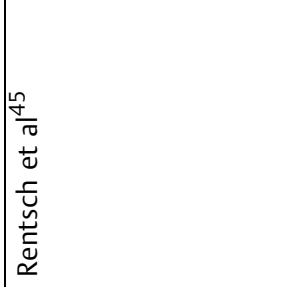 \\
\hline
\end{tabular}



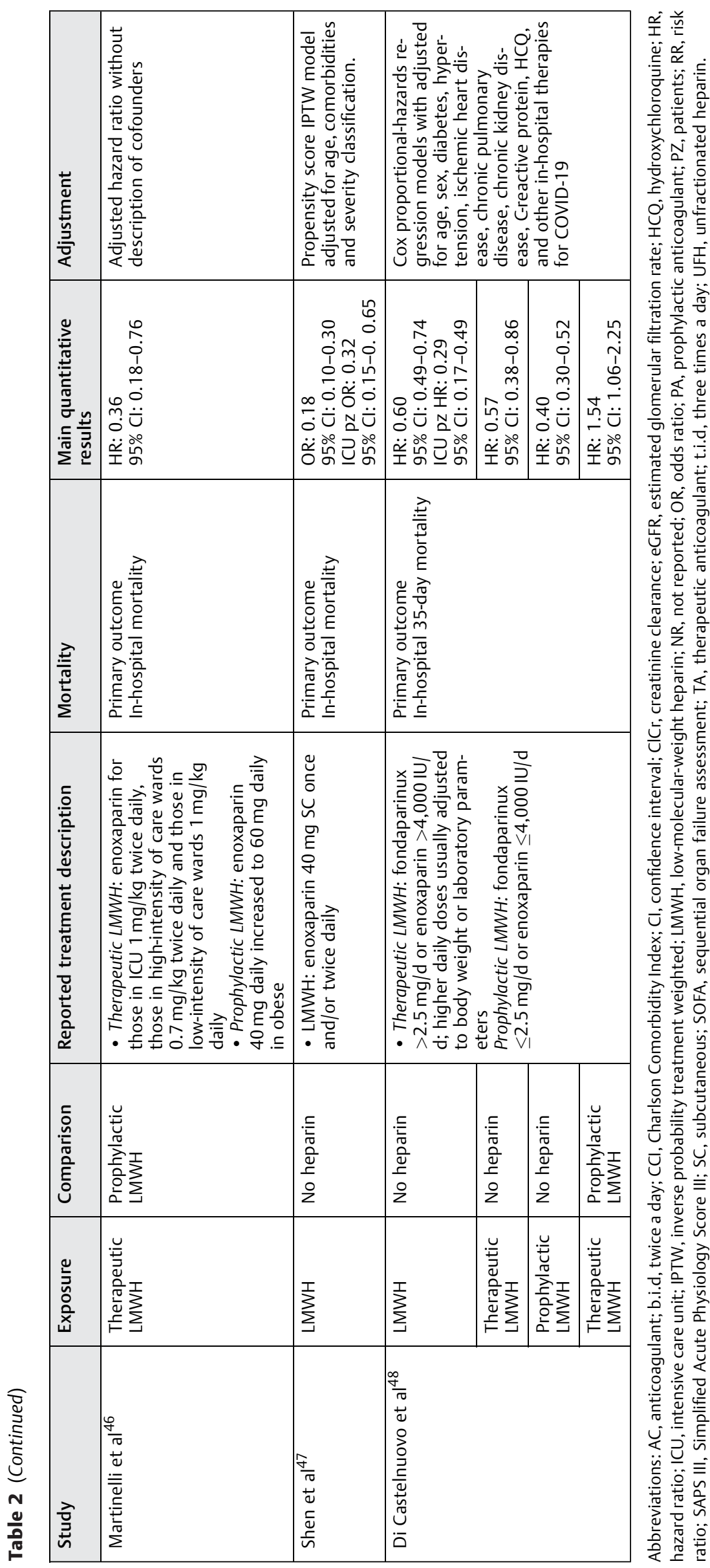
382 Anticoagulants, Mortality, and Bleeding in COVID-19 Patients Parisi et al.

Table 3 Main conclusions and limitations of the 29 selected studies

\begin{tabular}{|c|c|c|c|}
\hline Study & Main conclusions & Limitations & $\begin{array}{l}\text { Newcastle-Ottawa } \\
\text { Score system }\end{array}$ \\
\hline Tang et $\mathrm{al}^{20}$ & $\begin{array}{l}+ \\
\text { No difference in the } 28 \text {-day mortality } \\
\text { was found between heparin users and } \\
\text { nonusers ( } 30.3 \text { vs. } 29.7 \% \text { ). } \\
\text { AC therapy mainly with LMWH } \\
\text { appears to be associated with better } \\
\text { prognosis in severe COVID-19 } \\
\text { patients meeting SIC criteria or with } \\
\text { markedly elevated D-dimer. }\end{array}$ & $\begin{array}{l}\text { Concomitant therapies of anti- } \\
\text { COVID-19 were not evaluated. } \\
\text { The cohort included only severe } \\
\text { COVID-19 patients. }\end{array}$ & 9 \\
\hline Liu et $\mathrm{al}^{21}$ & $\begin{array}{l}\text { - } \\
\text { Exposure to a high dose of heparin } \\
\text { may trigger further severe throm- } \\
\text { bocytopenia with a fatal outcome. } \\
\text { An alternative anticoagulant other } \\
\text { than heparin should be used to treat } \\
\text { COVID-19 patients in critical } \\
\text { condition. }\end{array}$ & $\begin{array}{l}\text { Not peer reviewed. } \\
\text { Small sample size. } \\
\text { Dosage of treatment is not } \\
\text { reported. } \\
\text { Mortality was not primary } \\
\text { outcome. }\end{array}$ & 3 \\
\hline 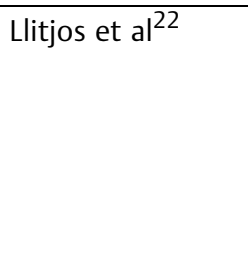 & $\begin{array}{l}\text { - } \\
\text { High rate of thromboembolic } \\
\text { events in COVID-19 patients treated } \\
\text { with therapeutic anticoagulation. } \\
\text { Our results support to consider } \\
\text { routine screening of VTE in severe } \\
\text { ICU COVID-19 patients. }\end{array}$ & $\begin{array}{l}\text { Small sample size. } \\
\text { Definitions of therapeutic and pro- } \\
\text { phylactic heparin doses are not } \\
\text { reported. } \\
\text { Mortality was not primary } \\
\text { outcome. }\end{array}$ & 3 \\
\hline Ayerbe et $\mathrm{al}^{23}$ & $\begin{array}{l}++ \\
\text { The administration of heparin was } \\
\text { associated with lower mortality in } \\
\text { patients admitted with COVID-19 }\end{array}$ & $\begin{array}{l}\text { Type and dosage of treatment not } \\
\text { reported. } \\
\text { Assessment of the outcome not } \\
\text { specified. }\end{array}$ & 9 \\
\hline Trinh et $\mathrm{al}^{24}$ & $\begin{array}{l}++ \\
\text { Therapeutic anticoagulation is as- } \\
\text { sociated with a survival advantage } \\
\text { among patients with COVID- } 19 \text { who } \\
\text { require mechanical ventilation in } \\
\text { ICU. } \\
\text { There was a trend toward increased } \\
\text { risk of bleeding in the TA group. }\end{array}$ & $\begin{array}{l}\text { Not peer reviewed. } \\
\text { Assessment of the outcome not } \\
\text { specified. }\end{array}$ & 8 \\
\hline Tremblay et al ${ }^{25}$ & $\begin{array}{l}\text { Our results suggest that AC alone is } \\
\text { unlikely to be protective for COVID- } \\
\text { 19-related morbidity and mortality. }\end{array}$ & $\begin{array}{l}\text { The cohort included both ambula- } \\
\text { tory and hospitalized patients. } \\
\text { Type and dosage of AC not } \\
\text { reported. }\end{array}$ & 8 \\
\hline Paranjpe et $a^{26}$ & $\begin{array}{l}++ \\
\text { Our findings suggest that systemic } \\
\text { AC may be associated with im- } \\
\text { proved outcomes (including mor- } \\
\text { tality) among patients hospitalized } \\
\text { with COVID-19. }\end{array}$ & $\begin{array}{l}\text { Type and dosage of AC not } \\
\text { reported. }\end{array}$ & 5 \\
\hline Al-Samkari et al ${ }^{27}$ & $\begin{array}{l}-1+ \\
\text { Receipt of therapeutic anticoagula- } \\
\text { tion early after ICU admission did } \\
\text { not affect survival. }\end{array}$ & $\begin{array}{l}\text { Definitions of therapeutic and pro- } \\
\text { phylactic dosages of heparin are not } \\
\text { reported. } \\
\text { Type of heparin not reported. }\end{array}$ & 6 \\
\hline Pesavento et al ${ }^{28}$ & $\begin{array}{l}\text { - } \\
\text { The subtherapeutic dose had a } \\
\text { higher incidence rate of mortality } \\
\text { than the prophylactic one. } \\
\text { - } \\
\text { In addition, the higher doses of } \\
\text { anticoagulants simultaneously in- } \\
\text { creased the bleeding events in both } \\
\text { MB and CRNMB. }\end{array}$ & $\begin{array}{l}\text { Mortality was not primary out- } \\
\text { come. } \\
\text { Risk analysis was not performed. } \\
\text { There is not a control group without } \\
\text { exposure. }\end{array}$ & 7 \\
\hline
\end{tabular}


Table 3 (Continued)

\begin{tabular}{|c|c|c|c|}
\hline Study & Main conclusions & Limitations & $\begin{array}{l}\text { Newcastle-Ottawa } \\
\text { Score system }\end{array}$ \\
\hline Russo et $\mathrm{al}^{29}$ & $\begin{array}{l}-1+ \\
\text { Preadmission anticoagulant treat- } \\
\text { ment did not affect the risk of death } \\
\text { during hospitalization in patients } \\
\text { with COVID-19. }\end{array}$ & $\begin{array}{l}\text { Anticoagulant treatment is consid- } \\
\text { ered in preadmission context. } \\
\text { Type and dosage of treatment are } \\
\text { not reported. } \\
\text { Mortality was not primary } \\
\text { outcome. }\end{array}$ & 5 \\
\hline Ferguson et $\mathrm{al}^{30}$ & $\begin{array}{l}+1- \\
\text { Therapeutic anticoagulant did not } \\
\text { improve the } 28 \text {-day mortality when } \\
\text { compared with the prophylactic dose. } \\
- \\
\text { Patients who received therapeutic } \\
\text { anticoagulation experienced five epi- } \\
\text { sodes of clinically apparent bleeding. } \\
\text { Those who received prophylactic dose } \\
\text { anticoagulation experienced four } \\
\text { episodes of clinically apparent } \\
\text { bleeding. }\end{array}$ & $\begin{array}{l}\text { Adjustments of analyses not } \\
\text { reported. } \\
\text { Concomitant therapies were not } \\
\text { evaluated. }\end{array}$ & 8 \\
\hline Schiavone et $\mathrm{al}^{31}$ & $\begin{array}{l}+ \\
\text { The use of heparin was associated } \\
\text { with a better chance of survival to } \\
\text { hospital discharge in COVID-19 } \\
\text { patients. }\end{array}$ & $\begin{array}{l}\text { Type and dosage of treatment are } \\
\text { not reported. } \\
\text { Adjustments of analyses not } \\
\text { reported. }\end{array}$ & 6 \\
\hline Desai et $a^{32}$ & $\begin{array}{l}++ \\
\text { Treatment with LMWH was found to } \\
\text { be protective in COVID-19-hospi- } \\
\text { talized patients. }\end{array}$ & $\begin{array}{l}\text { Dosage of anticoagulant is not } \\
\text { reported } \\
\text { Small sample size. }\end{array}$ & 9 \\
\hline Hsu et $\mathrm{al}^{33}$ & $\begin{array}{l}- \\
\text { The 30-day mortality was signifi- } \\
\text { cantly lower among all patients who } \\
\text { received high-intensity thrombo- } \\
\text { prophylaxis vs. those who received } \\
\text { standard prophylaxis. } \\
+ \text { /- } \\
\text { Patients who initially received high- } \\
\text { intensity prophylaxis or therapeutic } \\
\text { anticoagulation had improved } 30- \\
\text { day mortality without increased } \\
\text { rates of bleeding. }\end{array}$ & $\begin{array}{l}\text { Adjustments of analyses not } \\
\text { reported. } \\
\text { Small sample size. }\end{array}$ & 9 \\
\hline Gonzalez-Porras et al ${ }^{34}$ & $\begin{array}{l}++ \\
\text { The administration of LMWH at the } \\
\text { time of admission significantly re- } \\
\text { duced the mortality rate in unse- } \\
\text { lected adult COVID-19 patients. } \\
\text { Moreover, the magnitude of the } \\
\text { benefit was greater for the group of } \\
\text { patients who received high-dose } \\
\text { heparin. } \\
\text { - } \\
\text { Of note, the overall major bleeding } \\
\text { rate was more frequently reported } \\
\text { in the high-dose group, but only one } \\
\text { fatal event was reported. }\end{array}$ & $\begin{array}{l}\text { Not peer reviewed. } \\
\text { Adjustments of analyses not } \\
\text { reported. }\end{array}$ & 9 \\
\hline Albani et al $^{35}$ & $\begin{array}{l}++ \\
\text { Treatment with enoxaparin is asso- } \\
\text { ciated with a reduced mortality in } \\
\text { patients admitted to our hospital } \\
\text { with diagnosis of COVID-19, com- } \\
\text { pared with no enoxaparin } \\
\text { treatment. }\end{array}$ & - & 9 \\
\hline
\end{tabular}


384 Anticoagulants, Mortality, and Bleeding in COVID-19 Patients Parisi et al.

Table 3 (Continued)

\begin{tabular}{|c|c|c|c|}
\hline Study & Main conclusions & Limitations & $\begin{array}{l}\text { Newcastle-Ottawa } \\
\text { Score system }\end{array}$ \\
\hline lonescu et $\mathrm{al}^{36}$ & $\begin{array}{l}++ \\
\text { Both prophylactic and therapeutic } \\
\text { ACs were associated with decreased } \\
\text { mortality in COVID- } 19 \text {. } \\
\text { Patients receiving therapeutic doses } \\
\text { had higher survival probability com- } \\
\text { pared with those receiving prophy- } \\
\text { lactic doses, and the greatest effect } \\
\text { was observed in critically ill patients. } \\
- \\
\text { Major bleeding events occurred more } \\
\text { frequently in patients receiving TA. }\end{array}$ & $\begin{array}{l}\text { Precise indication for the initiation } \\
\text { of therapeutic AC was not available. } \\
\text { Patients treated with therapeutic } \\
\text { dose less than } 3 \text { days were included } \\
\text { in the prophylactic group TA in the } \\
\text { PA group. }\end{array}$ & 8 \\
\hline Nadkarni et al ${ }^{37}$ & $\begin{array}{l}++ \\
\text { Both therapeutic and prophylactic } \\
\text { anticoagulant groups had a reduced } \\
\text { in-hospital mortality compared } \\
\text { with no anticoagulation. } \\
\text { Therapeutic AC was associated with } \\
\text { a nonsignificant } 14 \% \text { reduction in } \\
\text { hazard of mortality compared with } \\
\text { prophylactic AC. } \\
- \\
\text { The proportion of patients with } \\
\text { bleeding events after initiation of } \\
\text { AC treatment was highest in } \\
\text { patients on therapeutic AC as } \\
\text { compared with patients on pro- } \\
\text { phylactic AC and no AC. }\end{array}$ & $\begin{array}{l}\text { Discrepancies between regimens of } \\
\text { treatment wherein doses may not } \\
\text { have accurately represented thera- } \\
\text { peutic and prophylactic AC. } \\
\text { Patients who were on both thera- } \\
\text { peutic and prophylactic doses of AC } \\
\text { were excluded due to inability to } \\
\text { definitively categorize them. }\end{array}$ & 9 \\
\hline Lynn et $\mathrm{al}^{38}$ & $\begin{array}{l}\text { - } \\
\text { Increased mortality was associated } \\
\text { with therapeutic AC compared with } \\
\text { prophylactic AC. } \\
\text { Approximately } 9 \% \text { of patients re- } \\
\text { ceiving therapeutic AC experienced } \\
\text { clinically significant bleeding or } \\
\text { thrombocytopenia, vs. 3\% in those } \\
\text { receiving prophylactic AC. }\end{array}$ & $\begin{array}{l}\text { Dosage of treatment is not fully } \\
\text { reported. } \\
\text { Adjusted analyses not reported. } \\
\text { Small sample size. }\end{array}$ & 5 \\
\hline Billett et $\mathrm{al}^{39}$ & $\begin{array}{l}++ \\
\text { COVID-19 patients with moderate } \\
\text { or severe illness benefit from anti- } \\
\text { coagulation showing a decreased } \\
\text { mortality. } \\
\text { There was no increase in transfusion } \\
\text { requirement with any of the anti- } \\
\text { coagulants used. }\end{array}$ & $\begin{array}{l}\text { The bleeding outcome was consid- } \\
\text { ered as transfusion requirement } \\
\text { and this does not take into account } \\
\text { the intracranial } \\
\text { or critical-site bleeds that would not } \\
\text { necessarily entail transfusion support. } \\
\text { Assessment of the outcome not } \\
\text { specified. }\end{array}$ & 9 \\
\hline Bolzetta et al ${ }^{40}$ & $\begin{array}{l}+ \text { l- } \\
\text { Therapeutic doses were not associ- } \\
\text { ated to a better survival rate. } \\
\text { In older people affected by COVID- } \\
19 \text { there is no justification for using } \\
\text { therapeutic doses instead of pro- } \\
\text { phylactic ones, having a similar im- } \\
\text { pact on mortality risk }\end{array}$ & $\begin{array}{l}\text { Dosage of treatment is not } \\
\text { reported. } \\
\text { Small sample size. } \\
\text { Assessment of the outcome not } \\
\text { specified. }\end{array}$ & 9 \\
\hline Falcone et al ${ }^{41}$ & $\begin{array}{l}++ \\
\text { LMWH was associated with a re- } \\
\text { duced risk of } 30 \text {-day mortality. } \\
- \\
\text { All patients who developed a major } \\
\text { bleeding received therapeutic dos- } \\
\text { ages of LMWH. }\end{array}$ & $\begin{array}{l}\text { Small sample size. } \\
\text { Among patients in the not treated } \\
\text { group, } 5 \text { of them were treated with } \\
\text { NOAC. } \\
\text { Patients at different dosages of } \\
\text { LMWH were considered together in } \\
\text { the analysis. }\end{array}$ & 9 \\
\hline
\end{tabular}


Table 3 (Continued)

\begin{tabular}{|c|c|c|c|}
\hline Study & Main conclusions & Limitations & $\begin{array}{l}\text { Newcastle-Ottawa } \\
\text { Score system }\end{array}$ \\
\hline Qin et $\mathrm{al}^{42}$ & $\begin{array}{l}++ \\
\text { LMWH emerged as an independent } \\
\text { factor for decreased 28-day } \\
\text { mortality. }\end{array}$ & $\begin{array}{l}\text { Adjustments of analyses not } \\
\text { reported. } \\
\text { Small sample size. } \\
\text { Among patients starting LMWH for } \\
\text { prophylaxis, } 19 \text { switched to thera- } \\
\text { peutic during the treatment period }\end{array}$ & 7 \\
\hline Jonmarker et al ${ }^{43}$ & $\begin{array}{l}++ \\
\text { Among critically ill COVID-19 } \\
\text { patients, high-dose thrombopro- } \\
\text { phylaxis was associated with a lower } \\
\text { risk of death. }\end{array}$ & $\begin{array}{l}\text { Small sample size. } \\
\text { Patients with chronic AC at admis- } \\
\text { sion, for reasons different from DVT } \\
\text { or PE, were included in the study }\end{array}$ & 9 \\
\hline Canoglu and Saylan ${ }^{44}$ & $\begin{array}{l}++ \\
\text { Mortality was higher in the pro- } \\
\text { phylactic group compared with the } \\
\text { therapeutic one. }\end{array}$ & $\begin{array}{l}\text { Small sample size. } \\
\text { No information on bleeding com- } \\
\text { plications } \\
\text { Different doses of LMWH used in } \\
\text { different clinics of the same } \\
\text { hospital. }\end{array}$ & 9 \\
\hline Rentsch et al ${ }^{45}$ & $\begin{array}{l}++ \\
\text { Early initiation of prophylactic anti- } \\
\text { coagulation among patients hospi- } \\
\text { talized with COVID-19 was } \\
\text { associated with a decreased risk of } \\
\text { mortality. }\end{array}$ & $\begin{array}{l}\text { Not peer reviewed. } \\
\text { The } 93 \% \text { of cohort is represented by } \\
\text { men. }\end{array}$ & 9 \\
\hline Martinelli et al ${ }^{46}$ & $\begin{array}{l}++ \\
\text { The cumulative incidence rate of } \\
\text { death was lower in patients treated } \\
\text { with high enoxaparin doses than in } \\
\text { those with the standard dose. } \\
- \\
\text { Four patients of the high enoxa- } \\
\text { parin dose had major bleeding } \\
\text { events. No bleeding event was ob- } \\
\text { served in the standard dosage pro- } \\
\text { phylaxis group. }\end{array}$ & $\begin{array}{l}\text { Small sample size. } \\
\text { Different types of therapeutic dos- } \\
\text { age according to different types of } \\
\text { patients (ICU, high-intensity and } \\
\text { low-intensity care ward). }\end{array}$ & 9 \\
\hline Shen et $\mathrm{al}^{47}$ & $\begin{array}{l}++ \\
\text { Among hospitalized COVID-19 } \\
\text { patients, LMWH use was associated } \\
\text { with lower all-cause in-hospital } \\
\text { mortality than } \\
\text { non-LMWH users. The survival ben- } \\
\text { efit was particularly significant } \\
\text { among more severely ill patients. }\end{array}$ & $\begin{array}{l}\text { Small sample size. } \\
\text { Two different dosages considered } \\
\text { together. }\end{array}$ & 9 \\
\hline Di Castelnuovo et al ${ }^{48}$ & $\begin{array}{l}++ \\
\text { The heparin use was associated with } \\
\text { lower mortality in hospitalized } \\
\text { COVID-19 patients }\end{array}$ & $\begin{array}{l}\text { Timing of the first dose of heparin at } \\
\text { admission and duration of treat- } \\
\text { ment could not be provided by } \\
\text { some clinical centers. } \\
\text { Specific reasons why patients were } \\
\text { treated or not with heparin could } \\
\text { not be collected }\end{array}$ & 9 \\
\hline
\end{tabular}

Abbreviations: AC, anticoagulant; CRNMB, clinical relevant non major bleeding; DVT, deep vein thrombosis; ICU, intensive care unit; LMWH, lowmolecular-weight heparin; MB, major bleeding; NOAC, non-vitamin K oral anticoagulant; PA, prophylactic anticoagulant; SIC, sepsis-induced coagulopathy; TA, therapeutic anticoagulant; VTE, venous thromboembolism. 
On the contrary, Gonzalez-Porras et al and Martinelli et al demonstrated that the benefit of the administration of LMWH on in-hospital mortality was higher for the groups receiving the higher doses. ${ }^{34,46}$ The study by Nadkarni et al reported a not statistically significant reduction of in-hospital mortality risk, when therapeutic anticoagulant treatment was associated with the prophylactic regimen (HR: 0.86, 95\% CI: 0.73-1.02; - Table 2). ${ }^{37}$ Finally, Bolzetta et al indicated that in a cohort of elderly affected by COVID-19, there was no justification for using therapeutic instead of prophylactic doses, having a similar impact on in-hospital mortality risk (HR: 0.89, 95\% CI: 0.30-2.71) (-Table 2). ${ }^{40}$

The five studies that included only ICU patients showed opposite findings, $22,24,30,43,44$ and two of them were of low quality (-Supplementary Table S1). The small study of Llitjos et al did not consider overall mortality as a primary outcome; however, it reported the same incident rate in both heparin dosage treatment groups, but the therapeutic dose of heparin (LMWH or UFH) resulted in a higher rate of thromboembolic events in COVID-19 patients. ${ }^{22}$ On the contrary, Trinh et al (a non-peer-reviewed study), Jonmarker et al, and Canoglu and Saylan observed that therapeutic anticoagulation was associated with survival advantage among ICU patients with COVID-19. ${ }^{24,43,44}$ Finally, the study by Ferguson et al reported that therapeutic anticoagulation did not improve mortality at 28 days compared with the prophylactic dosage (HR: 0.73, 95\% CI: 0.33-1.76). ${ }^{30}$

\section{Qualitative Review: Anticoagulant Use and Bleeding in COVID-19 Patients}

Several studies reported incidence of different types of bleeding (gastrointestinal, intracranial, mucocutaneous, and bronchopulmonary) which occurred during the hospitalization period of COVID-19 patients treated with anticoagulants. ${ }^{24,28,30,33,34,36-38,41-43,46,47}$ The majority of the articles reported that treatment with a therapeutic/ higher dosage of anticoagulants was associated with a higher incidence of bleeding. ${ }^{28,30,34,36-38,41,46}$ Qin et al observed that occurrence of bleeding events was higher in the group treated with LMWH compared with the nontreated. ${ }^{42}$ In addition, the study by Trinh et al showed that there was a trend toward increased risk of bleeding in the therapeutic group. ${ }^{24}$ On the other hand, the study by Hsu et al showed that there was no difference in the incidence of bleeding events between therapeutic and prophylactic groups. ${ }^{33}$ In addition, Jonmarker et al reported that bleeding events occurred more frequently in the low LMWH dose group $(11.9 \%)$ than in the high-dose group (2.7\%), although the findings were not statistically significant $(p=0.16){ }^{43}$

\section{Quantitative Meta-Analysis}

Of the 29 selected studies mentioned above, 16 were included in the main, quantitative meta-analysis (anticoagulant use vs. no anticoagulant use). ${ }^{20,23,27,31-37,39,41,42,45,47,48}$ A secondary analysis based on 10 studies $24,30,33,34,37,40,43,44,46,48$ was performed to compare different dosages of anticoagulants (therapeutic vs. prophylactic). In addition, we separately investigated the associa- tion of prophylactic and therapeutic anticoagulant regimens with in-hospital mortality, compared with the nontreated control group.

The studies by Liu et al, Llitjos et al, Pesavento et al, and Lynn et al were excluded because the adjusted associations of anticoagulant use with in-hospital all-cause mortality were not reported. ${ }^{21,22,28,38}$ The study by Paranjpe et $\mathrm{al}^{26}$ was excluded as part of another study already included. ${ }^{37}$ Since the study by Tremblay et al $^{25}$ included both outpatients and hospitalized patients and the report by Russo et $\mathrm{al}^{29}$ considered anticoagulant treatment only in the preadmission context, they were both excluded from our meta-analyses.

-Fig. 1 shows that by pooling all the 16 selected studies, the use of anticoagulant was associated with a reduced inhospital all-cause mortality risk of 50\% (pooled RR: $0.50,95 \%$ CI: $0.40-0.62$; high level of heterogeneity: $I^{2}$ : $87 \%$, random effects model). Results from fixed effects analysis are reported in -Supplementary Fig. S3 (pooled RR: 0.60, 95\% CI: $\left.0.56-0.64 ; I^{2:} 87 \%\right)$.

By pooling the 14 studies on all hospitalized COVID-19 patients, which accounted for $86.1 \%$ of the total weight (-Fig. 1), a 55\% lower in-hospital all-cause mortality risk was found (pooled RR: 0.45, 95\% CI: 0.37-0.54; high level of heterogeneity: $I^{2}: 76 \%$, random effects model); on the contrary, the subgroup meta-analysis considering ICU or severe patients showed no association between anticoagulant treatment and in-hospital all-cause mortality (13.9\% of the weight; pooled RR: $1.23,95 \% \mathrm{CI}$ : 0.89-1.71; medium level of heterogeneity: $I^{2}: 36 \%$, random effects model). The latter finding was confirmed by including data on ICU patients from Shen et al and Di Castelnuovo et al's studies (pooled RR: 0.66, 95\% CI: 0.30-1.45; $I^{2}$ : 91\%, random effect; -Supplementary Table S2 and -Supplementary Fig. S4). ${ }^{47,48}$

In the "Meta-Analysis: Data Extraction and Data Analysis" section, we described that three of the selected studies separately reported the association with in-hospital mortality for both anticoagulant regimens and data on prophylactic dosage were extracted and considered for the main metaanalysis. Nevertheless, findings did not change when data on therapeutic regimen of these three studies were considered (pooled RR: 0.49, 95\% CI: 0.39-0.62; high level of heterogeneity: $I^{2}$ : 90\%, random effects model; -Supplementary Fig. S5). Additionally, in a further sensitivity analysis, the inclusion of nonadjusted estimate from one study originally excluded $^{21}$ did not modify the result (-Supplementary Table S2 and - Supplementary Fig. S6).

In comparison with no anticoagulant use, both treatments at prophylactic and therapeutic doses were found associated with a $58 \%$ (pooled RR: $0.42,95 \% \mathrm{CI}: 0.37-0.47 ; I^{2}$ : $0 \%$; -Supplementary Table S2 and -Supplementary Fig. S7) and 43\% (pooled RR: 0.57, 95\% CI: 0.38-0.86; $I^{2}$ : 93\%; -Supplementary Table S2 and -Supplementary Fig. S8) lower in-hospital all-cause mortality risk, respectively.

The subgroup analysis, including 11 studies reporting exclusively heparin (LMWH or UFH) treatment $(N=11,586)$, confirmed that the treated group had a reduced in-hospital all-cause mortality risk compared with the 
Anticoagulants, Mortality, and Bleeding in COVID-19 Patients Parisi et al. 387

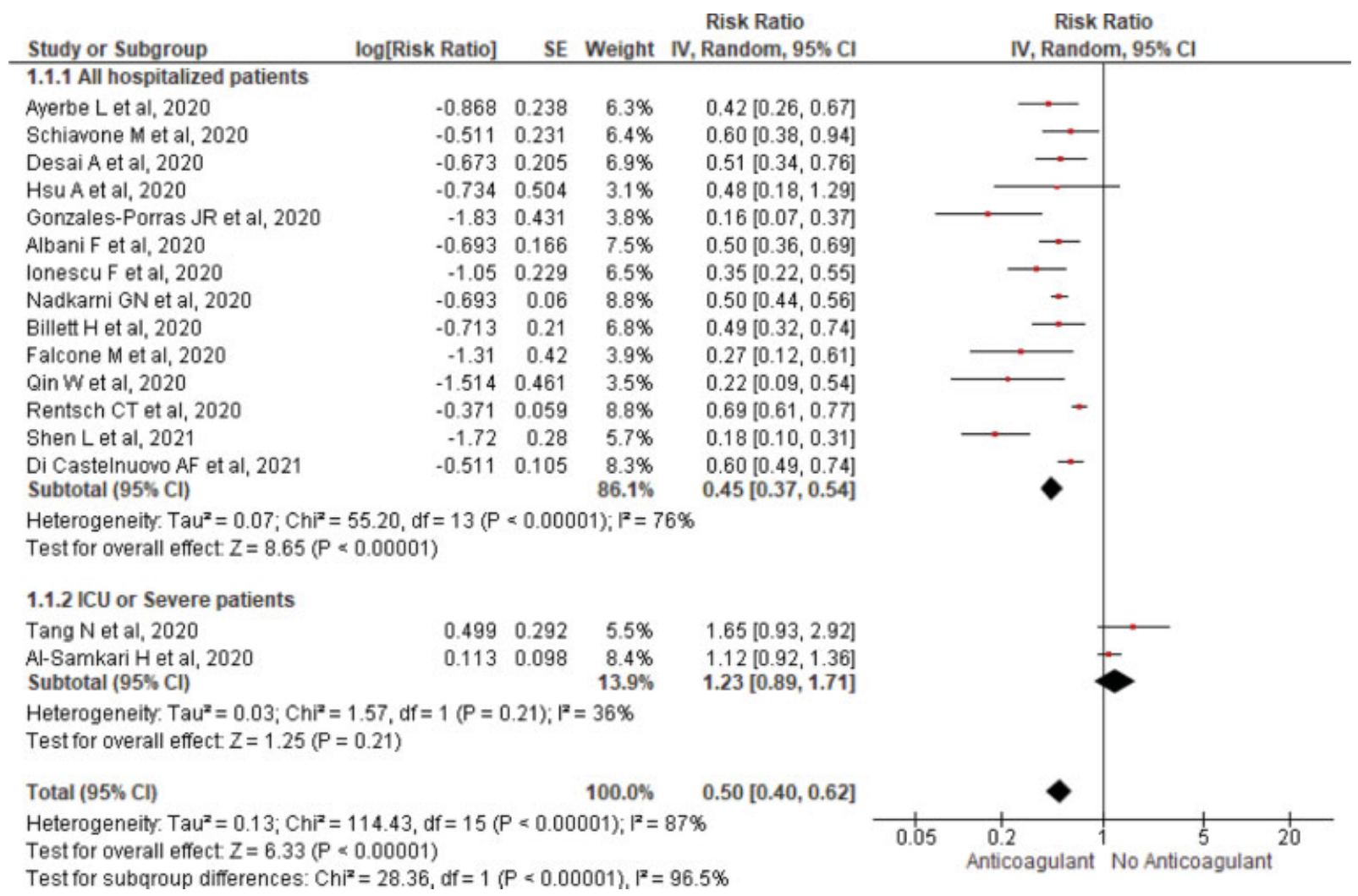

Fig. 1 Forest plot for association of anticoagulant use with in-hospital all-cause mortality in hospitalized COVID-19 patients $(N=25,719)$; random model.

control (pooled RR: 0.44, 95\% CI: 0.33-0.59; high level of heterogeneity: $I^{2}$ : 79\%, random effects model; - Fig. 2 and - Supplementary Table S2).

By pooling 10 studies on all hospitalized COVID-19 patients, a reduction of $43 \%$ in in-hospital all-cause mortality risk was found, when the therapeutic dosage was compared with the prophylactic dosage (pooled RR: $0.57,95 \% \mathrm{CI}$ : $0.38-0.86$; high level of heterogeneity: $I^{2}: 81 \%$, random effect). The previous finding resulted stronger in the subgroup analysis considering four studies on ICU or severe

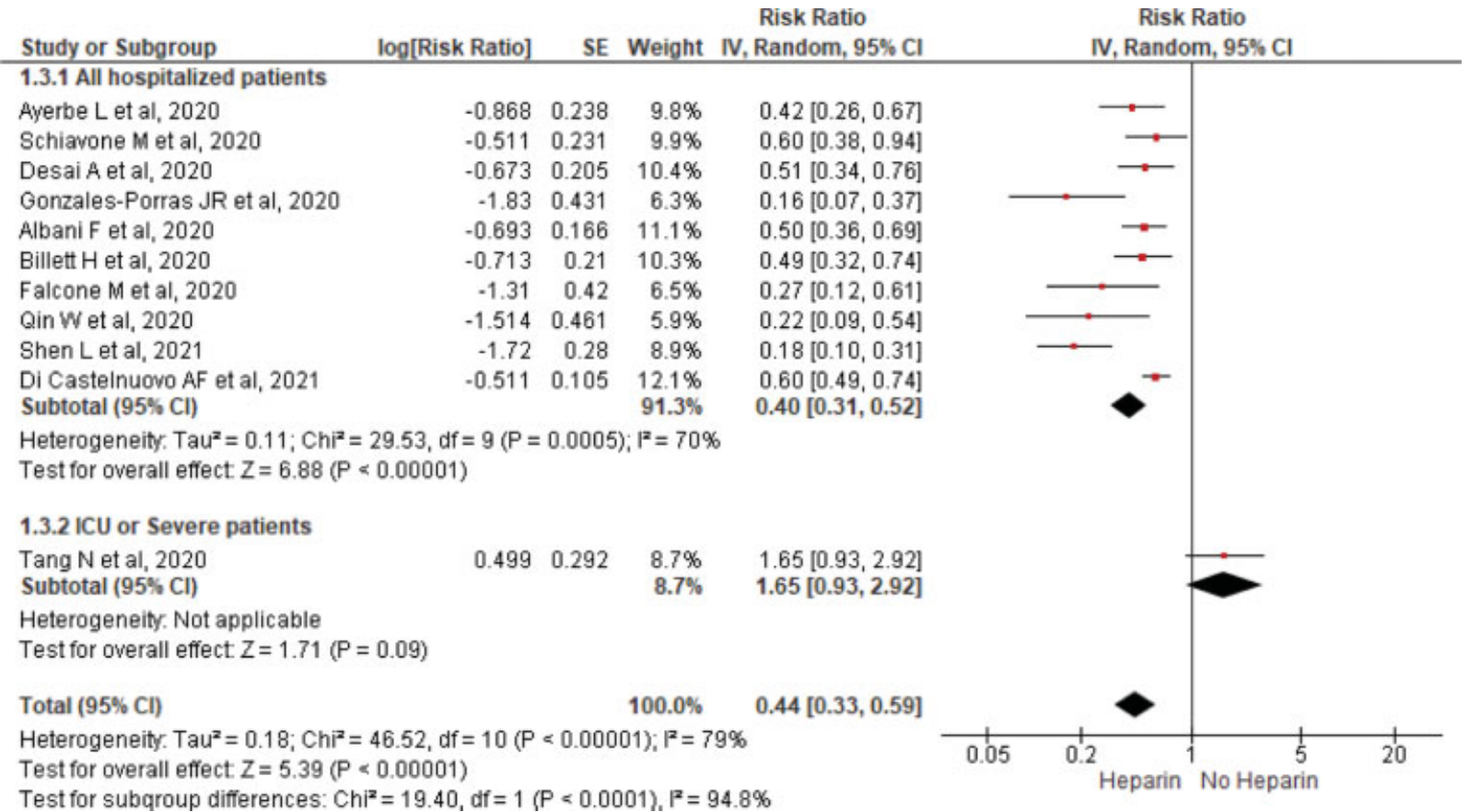

Fig. 2 Forest plot for association of heparin use with in-hospital all-cause mortality in hospitalized COVID-19 patients $(N=11,586)$; random model. 


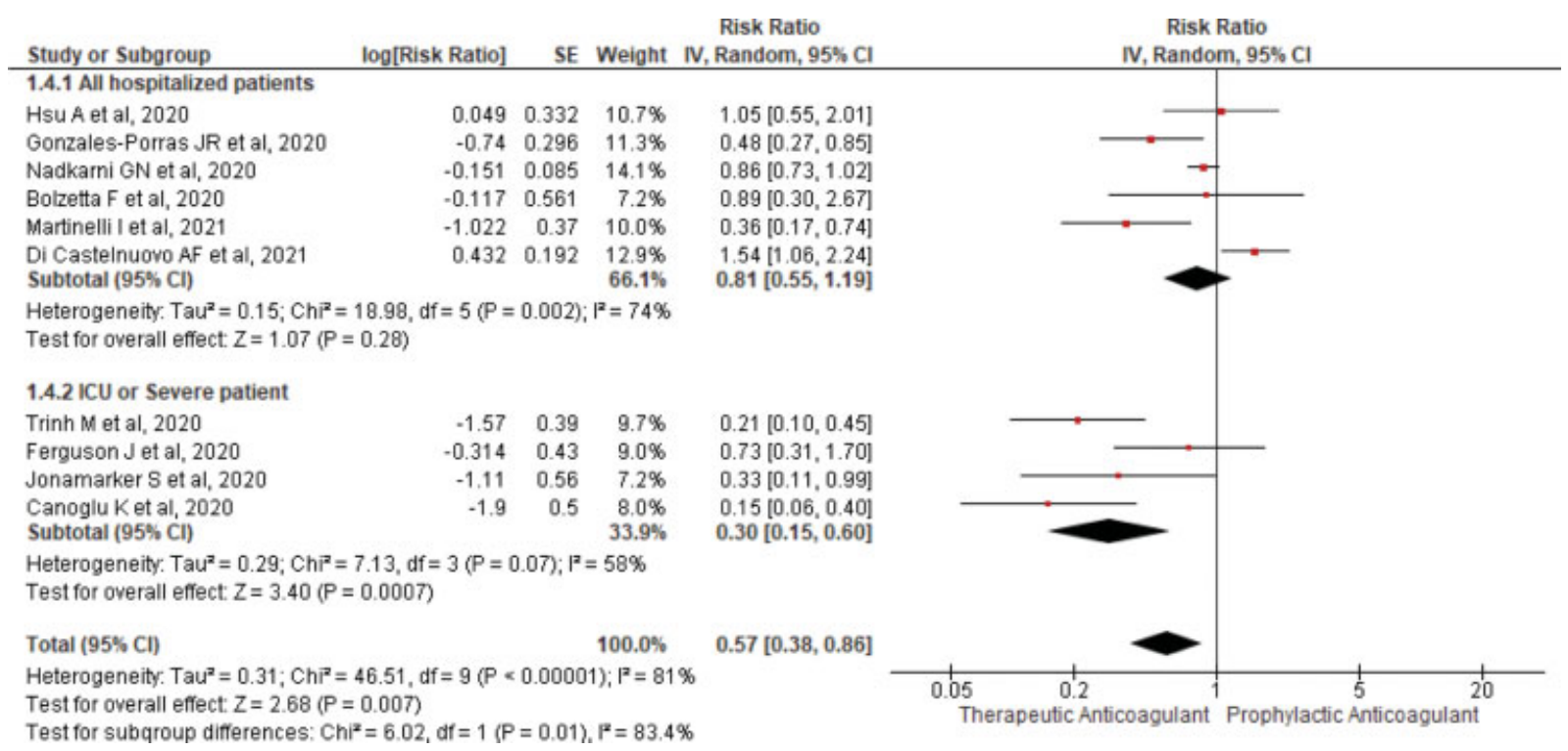

Fig. 3 Forest plot for association of two different dosages of anticoagulant (therapeutic vs. prophylactic) with in-hospital all-cause mortality in all hospitalized COVID-19 patients $(N=6,113)$; random model.

COVID-19 patients (pooled RR: $0.30,95 \% \mathrm{CI}: 0.15-0.60$; medium level of heterogeneity: $\mathrm{I}^{2}: 58 \%$ ) (-Fig. 3). Further inclusion of not adjusted studies did not change the latter finding (-Supplementary Fig. 59).

-Fig. 4 shows that the anticoagulant prophylactic dosage was not associated with bleeding in comparison with no use (pooled RR: 0.77, 95\% CI: 0.38-1.55; $I^{2}: 60 \%$, random effects model; panel A). On the contrary, the use of therapeutic doses of anticoagulant increased the risk of bleeding (pooled RR: $1.57,95 \%$ CI: $1.14-2.16 ; I^{2}$ : $0 \%$, random effects model; -Fig. 4, panel B), compared with nontreated COVID-19 patients. A further meta-analysis confirms that

Panel A.

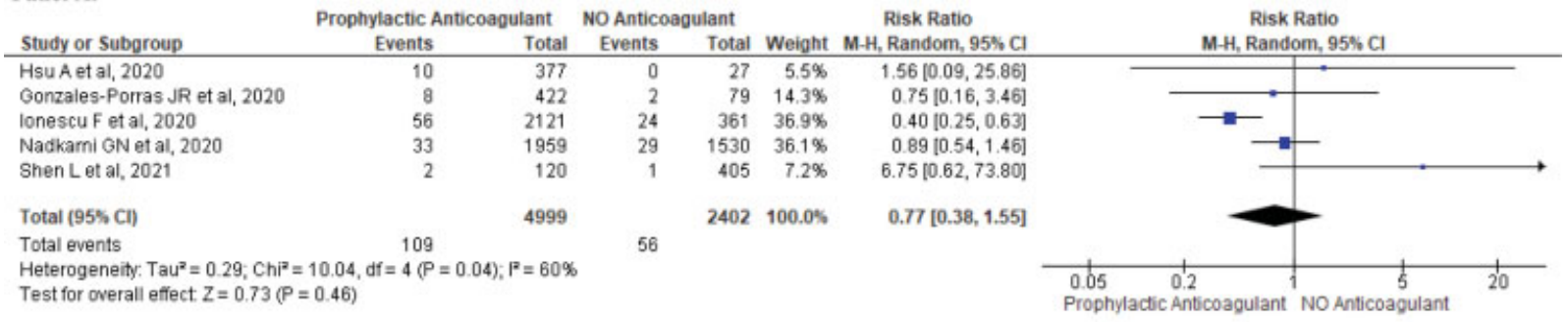

Panel B.

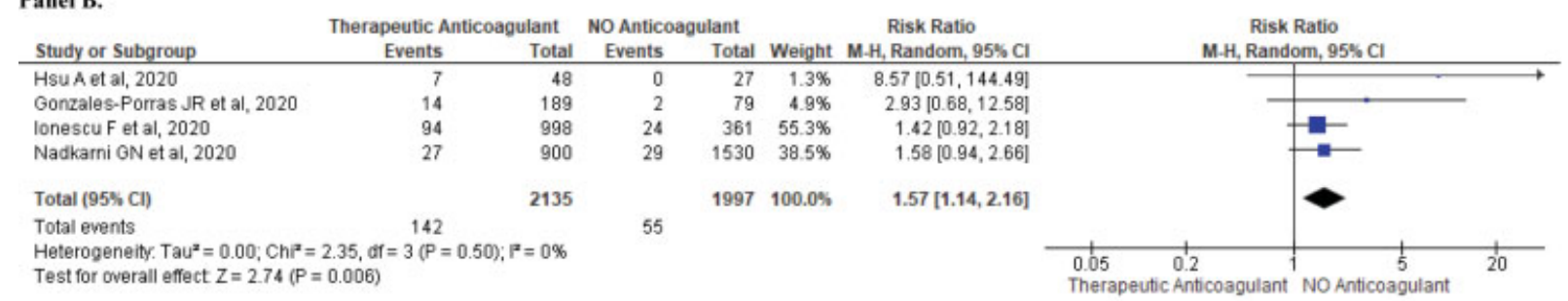

Panel C.

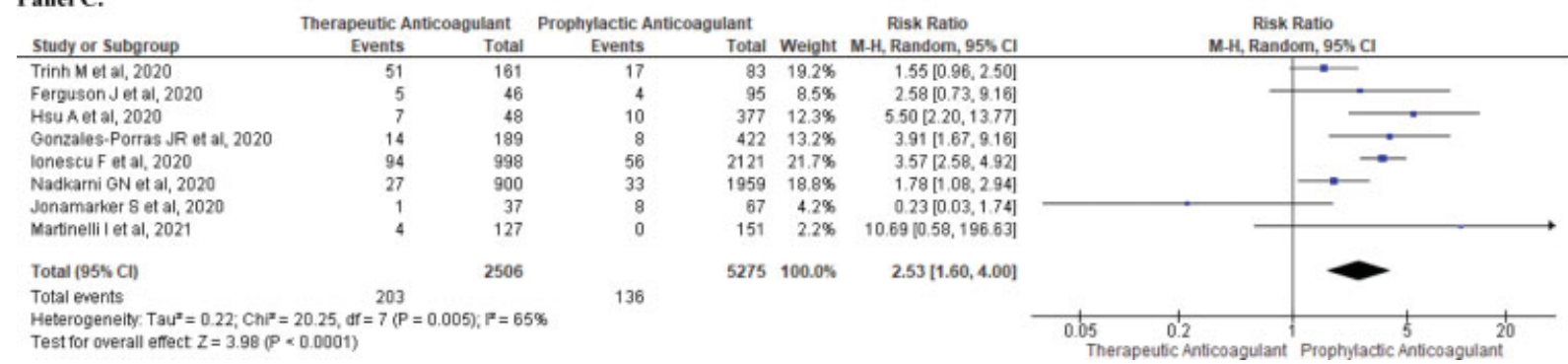

Fig. 4 Panel A: forest plot for association of prophylactic dosage of anticoagulants with bleeding occurrence in COVID-19 patients ( $N=7,401)$, random model. Panel B: forest plot for association of therapeutic dosage of anticoagulants with bleeding occurrence in COVID-19 patients $(N=4,132)$, random model. Panel C: forest plot for association of two different dosages of anticoagulant (therapeutic vs. prophylactic) with bleeding occurrence in all hospitalized COVID-19 patients $(N=7,781)$; random model. 
patients treated with therapeutic doses of anticoagulants were at a higher risk of bleeding (pooled RR: $2.53,95 \% \mathrm{CI}$ : 1.60-4.00; $I^{2}$ : 65\%, random effects model; - Fig. 4, panel C) compared with those at prophylactic dosages. Results from fixed effects analyses are reported in - Supplementary Fig. S10, panels A-C.

\section{Discussion}

The main finding from the present analyses is that anticoagulant use, mainly as heparin, was associated with a significantly lower risk of in-hospital all-cause mortality among hospitalized COVID-19 patients.

A still open question on the use of anticoagulation in COVID-19 patients is if therapeutic doses of anticoagulant are more effective than the low doses used as prophylactic. According to our findings, both anticoagulant regimens reduced in-hospital all-cause mortality in COVID-19 patients, although the therapeutic dosage did it to a greater degree than the prophylactic, particularly when ICU patients were considered. At the same time, the therapeutic dosages were found to be associated with a higher risk of bleeding. It is well known that exposure to high doses of anticoagulant could lead to the occurrence of bleeding events, often resulting in fatal outcome. ${ }^{12,14,15}$

The results of our meta-analyses are in line with the recommendations of major guidelines suggesting that all hospitalized COVID-19 patients, even those not in the ICU, should receive prophylactic doses of $\mathrm{LMWH}$, in the absence of contraindications. ${ }^{11-13}$

Recently, three meta-analyses investigated the effect of anticoagulation on in-hospital all-cause mortality in patients with COVID-19. ${ }^{51-53}$ The first two found that anticoagulant therapy (any dosage) was not associated with increased risk of mortality. Both meta-analyses included studies that did not meet our inclusion criteria. ${ }^{25,29,54-57}$ In particular, the meta-analysis by Lu et al, among the five selected studies $(N=8,533)$, included two studies reporting the effect of anticoagulant treatment in a preadmission context. However, the exclusion of these two studies ${ }^{25,29}$ did not change the results (RR: $0.79,95 \% \mathrm{CI}: 0.48-1.31) .{ }^{51}$ On the other hand, Salah et al used nonadjusted estimates in their meta-analysis (six studies, $N=6,390) .^{52}$

Finally, our results are in line with recent findings by Kamel et al that showed a favorable effect of in-hospital anticoagulant treatment on in-hospital mortality in COVID19 patients (RR:0.56, 95\% CI: 0.36-0.92, five studies, $N=4,229$ ). Additionally, they reported that the prophylactic dose might be associated with higher in-hospital mortality than the therapeutic anticoagulant (RR: 1.58, 95\% CI: $1.34-1.87$, three studies, $N=963) .{ }^{53}$ We performed sensitivity analyses according to type of COVID-19 patients (hospitalized or ICU patients) and on exclusive heparin treatment.

Conflicting results, due to the wide heterogeneity of the study setting, population, and therapeutic approaches, underline the urgent need for randomized controlled clinical trials to define the effect of anticoagulant dosages in patients with COVID-19. In addition, the major guidelines have not yet recommended a standardized protocol for the management of COVID-19 patients. The only exception is the position paper by the Italian Society on Thrombosis and Haemostasis that defined the prophylactic dose of LMWH as enoxaparin 4,000 IU subcutaneously every 12 hours. ${ }^{14}$ As a consequence, the only suggestions available for the choice of treatment in COVID-19 patients are based on the VTE risk stratification, the monitoring of specific laboratory parameters, (hemostasis function and platelet count), and the evaluation of the personal clinical history of each single patient. $^{14,16}$

\section{Strengths and Limitations}

The present article has the strength of including all relevant studies not included in previous reviews until now, ${ }^{27,36,37,39-48}$ analyzing a greater number of studies and of COVID-19 patients than those of previous studies. $^{51-53}$

Its major limitation is that all primary studies are observational, and that subgroup analyses suffer from a high degree of heterogeneity. In particular, prophylactic and therapeutic dosages were not defined in a standardized way, as well as the assessment of major or nonmajor clinically relevant bleeding complications. Our results should therefore be considered with caution, since the possibility of confounding could not be fully excluded.

\section{Conclusions}

We report a significant reduction of in-hospital all-cause mortality in COVID-19 patients treated with anticoagulants (mainly heparin). Both anticoagulant regimens are associated with a better survival in COVID-19 patients (therapeutic dosages at a higher extent than prophylactic), particularly in ICU patients. However, due to the higher risk of bleeding at therapeutic doses, in noncritically ill COVID-19 patients, the use of prophylactic dosages of anticoagulant is probably to be preferred.

Therefore, while waiting for definitive answers from the ongoing clinical trials, it is important, especially in this period of spread resurgence of the pandemic, to pay attention to the type and dosage of anticoagulant used in the management of hospitalized COVID-19 patients. Randomized controlled clinical trials will be necessary before any conclusion can be reached regarding a potential benefit of these drugs in patients with COVID-19.

\section{Authors' Contributions}

S.C. and L.I. contributed to the conception and design of the work and interpretation of data; R.P., S.C., and A.D.C. managed study selection and data extraction and critically reviewed the results; R.P. analyzed the data; R.P. and S.C. wrote the paper; L.I., G.d.G., and M.B.D. originally inspired the research and critically reviewed the manuscript. All authors approved the final version of the manuscript.

Funding

None. 


\section{Conflict of Interest}

The authors report no conflict of interest related to the current work. A.D.C. reports grants from ERAB (the European Foundation for Alcohol Research), outside the submitted work. Dr. Costanzo reports grants from ERAB (the European Foundation for Alcohol Research), personal fees from The Dutch Beer Institute foundation-The Brewers of Europe, outside the submitted work.

\section{Acknowledgments}

S.C. was the recipient of a Fondazione Umberto Veronesi Travel Grant.

\section{References}

1 Luo W, Yu H, Gou J, et al. Clinical pathology of critical patient with novel coronavirus pneumonia (COVID-19). Preprints 2020. Doi: 10.1097/TP.0000000000003412

2 Menter T, Haslbauer JD, Nienhold R, et al. Postmortem examination of COVID-19 patients reveals diffuse alveolar damage with severe capillary congestion and variegated findings in lungs and other organs suggesting vascular dysfunction. Histopathology 2020;77(02):198-209

3 Dolhnikoff M, Duarte-Neto AN, de Almeida Monteiro RA, et al. Pathological evidence of pulmonary thrombotic phenomena in severe COVID-19. J Thromb Haemost 2020;18(06):1517-1519

4 Middeldorp S, Coppens M, van Haaps TF, et al. Incidence of venous thromboembolism in hospitalized patients with COVID-19. J Thromb Haemost 2020;18(08):1995-2002

5 Abou-Ismail MY, Diamond A, Kapoor S, Arafah Y, Nayak L. The hypercoagulable state in COVID-19: Incidence, pathophysiology, and management. Thromb Res194:101-115

6 Bikdeli B, Madhavan MV, Jimenez D, et al; Global COVID-19 Thrombosis Collaborative Group, Endorsed by the ISTH, NATF, ESVM, and the IUA, Supported by the ESC Working Group on Pulmonary Circulation and Right Ventricular Function. COVID-19 and thrombotic or thromboembolic disease: implications for prevention, antithrombotic therapy, and follow-up: JACC stateof-the-art review. J Am Coll Cardiol 2020;75(23):2950-2973

7 Lucatelli P, De Rubeis G, Citone M, et al. Heparin-related major bleeding in covid-19-positive patient: perspective from the outbreak. Cardiovasc Intervent Radiol 2020;43(08): 1216-1217

8 Tang N, Li D, Wang X, Sun Z. Abnormal coagulation parameters are associated with poor prognosis in patients with novel coronavirus pneumonia. J Thromb Haemost 2020;18(04):844-847

9 Mycroft-West CJ, Su D, Pagani I, et al. Heparin inhibits cellular invasion by SARS-CoV-2: structural dependence of the interaction of the spike s1 receptor-binding domain with heparin. Thromb Haemost 2020;120(12):1700-1715

10 Hippensteel JA, LaRiviere WB, Colbert JF, Langouët-Astrié CJ, Schmidt EP. Heparin as a therapy for COVID-19: current evidence and future possibilities. Am J Physiol Lung Cell Mol Physiol 2020; 319(02):L211-L217

11 World Health Organization. Clinical management of severe acute respiratory infection (SARI) when COVID-19 disease is suspected. WHO/2019-nCoV/clinical/2020.4 2020. Accessed February 4, 2021 at: https://apps.who.int/iris/handle/10665/331446

12 CDC. Interim clinical guidance for management of patients with confirmed coronavirus disease (COVID-19). Published 2020. Updated December 8, 2020. Accessed January 8, 2021 at: https://www.cdc.gov/coronavirus/2019-ncov/hcp/clinical-guidance-management-patients.html

13 Matos R, Chung K. DoD COVID-19 practice management guide: clinical management of COVID-19. Published 2020. Updated March 23, 2020. Accessed September 28, 2020 at: https://asprtra- cie.hhs.gov/technical-resources/resource/7899/dod-covid-19practice-management-guide-clinical-management-of-covid-19

14 Marietta M, Ageno W, Artoni A, et al. COVID-19 and haemostasis: a position paper from Italian Society on Thrombosis and Haemostasis (SISET). Blood Transfus 2020;18(03):167-169

15 Thachil J, Tang N, Gando S, et al. ISTH interim guidance on recognition and management of coagulopathy in COVID-19. J Thromb Haemost 2020;18(05):1023-1026

16 Barnes GD, Burnett A, Allen A, et al. Thromboembolism and anticoagulant therapy during the COVID-19 pandemic: interim clinical guidance from the anticoagulation forum. J Thromb Thrombolysis 2020;50(01):72-81

17 Marietta M, Vandelli P, Mighali P, Vicini R, Coluccio V, D'Amico RCOVID-19 HD Study Group. Randomised controlled trial comparing efficacy and safety of high versus low low-molecular weight heparin dosages in hospitalized patients with severe COVID-19 pneumonia and coagulopathy not requiring invasive mechanical ventilation (COVID-19 HD): a structured summary of a study protocol. Trials 2020;21(01):574

18 Kharma N, Roehrig S, Shible AA, et al. Anticoagulation in critically ill patients on mechanical ventilation suffering from COVID-19 disease, The ANTI-CO trial: a structured summary of a study protocol for a randomised controlled trial. Trials 2020;21(01):769

19 Higgins JPT, Thomas J, Chandler J, et al. Cochrane Handbook for Systematic Reviews of Interventions version 6.0 (updated July 2019). Cochrane, 2019. Accessed February 4, 2021 at: www.training.cochrane.org/handbook

20 Tang N, Bai H, Chen X, Gong J, Li D, Sun Z. Anticoagulant treatment is associated with decreased mortality in severe coronavirus disease 2019 patients with coagulopathy. J Thromb Haemost 2020;18(05):1094-1099

21 Liu X, Zhang X, Xiao Y, et al. Heparin-induced thrombocytopenia is associated with a high risk of mortality in critical COVID-19 patients receiving heparin-involved treatment. MedRxiv 2020. Doi: $10.1101 / 2020.04 .23 .20076851$

22 Llitjos JF, Leclerc M, Chochois C, et al. High incidence of venous thromboembolic events in anticoagulated severe COVID-19 patients. J Thromb Haemost 2020;18(07):1743-1746

23 Ayerbe L, Risco C, Ayis S. The association between treatment with heparin and survival in patients with Covid-19. J Thromb Thrombolysis 2020;50(02):298-301

24 Trinh M, Chang DR, Govindarajulu US, et al. Therapeutic anticoagulation is associated with decreased mortality in mechanically ventilated COVID-19 patients. MedRxiv 2020. Doi: 10.1101/2020.05.30.20117929

25 Tremblay D, van Gerwen M, Alsen M, et al. Impact of anticoagulation prior to COVID-19 infection: a propensity scorematched cohort study. Blood 2020;136(01):144-147

26 Paranjpe I, Fuster V, Lala A, et al. Association of treatment dose anticoagulation with in-hospital survival among hospitalized patients with COVID-19. J Am Coll Cardiol 2020;76(01):122-124

27 Al-Samkari H, Gupta S, Karp Leaf R, et al. Thrombosis, bleeding, and the effect of anticoagulation on survival in critically ill patients with COVID-19 in the United States. Res Pract Thromb Haemost 2020;4(Suppl 1 Accessed July 17, 2020 at: https:// abstracts.isth.org/abstract/thrombosis-bleeding-and-the-effectof-anticoagulation-on-survival-in-critically-ill-patients-withcovid-19-in-the-united-states/

28 Pesavento R, Ceccato D, Pasquetto G, et al. The hazard of (sub) therapeutic doses of anticoagulants in non-critically ill patients with Covid-19: the Padua province experience. J Thromb Haemost 2020;18(10):2629-2635

29 Russo V, Di Maio M, Attena E, et al. Clinical impact of preadmission antithrombotic therapy in hospitalized patients with COVID-19: a multicenter observational study. Pharmacol Res 2020;159:104965

30 Ferguson J, Volk S, Vondracek T, Flanigan J, Chernaik A. Empiric therapeutic anticoagulation and mortality in critically ill patients 
with respiratory failure from SARS-CoV-2: a retrospective cohort study. J Clin Pharmacol 2020;60(11):1411-1415

31 Schiavone M, Gasperetti A, Mancone M, et al. Oral anticoagulation and clinical outcomes in COVID-19: An Italian multicenter experience. Int J Cardiol 2021;323:276-280

32 Desai A, Voza G, Paiardi S, et al. The role of anti-hypertensive treatment, comorbidities and early introduction of LMWH in the setting of COVID-19: a retrospective, observational study in Northern Italy. Int J Cardiol 2021;324:249-254

33 Hsu A, Liu Y, Zayac AS, Olszewski AJ, Reagan JL. Intensity of anticoagulation and survival in patients hospitalized with COVID-19 pneumonia. Thromb Res 2020;196:375-378

34 Gonzalez-Porras JR, Belhassen-Garcia M, Bernus AL, VaqueroRoncero LM. Low molecular weight heparin in adults inpatient COVID-19. Accessed February 4, 2021 at: SSRN: https:// ssrn.com/abstract $=3586665$

35 Albani F, Sepe L, Fusina F, et al. Thromboprophylaxis with enoxaparin is associated with a lower death rate in patients hospitalized with SARS-CoV-2 infection. A cohort study. EClinicalMedicine 2020;27:100562

36 Ionescu F, Jaiyesimi I, Petrescu I, et al. Association of anticoagulation dose and survival in hospitalized COVID-19 patients: a retrospective propensity score-weighted analysis. Eur J Haematol 2021;106(02):165-174

37 Nadkarni GN, Lala A, Bagiella E, et al. Anticoagulation, bleeding, mortality, and pathology in hospitalized patients with COVID-19. J Am Coll Cardiol 2020;76(16):1815-1826

38 Lynn L, Reyes JA, Hawkins K, et al. The effect of anticoagulation on clinical outcomes in novel coronavirus (COVID-19) pneumonia in a U.S. cohort. Thromb Res 2021;197:65-68

39 Billett HH, Reyes-Gil M, Szymanski J, et al. Anticoagulation in COVID-19: effect of enoxaparin, heparin, and apixaban on mortality. Thromb Haemost 2020;120(12):1691-1699

40 Bolzetta F, Maselli M, Formilan M, et al. Prophylactic or therapeutic doses of heparins for COVID-19 infection? A retrospective study. Aging Clin Exp Res 2021;33(01):213-217

41 Falcone M, Tiseo G, Barbieri G, Galfo V, Russo A, Virdis A. Role of low-molecular-weight heparin in hospitalized patients with severe acute respiratory syndrome coronavirus 2 pneumonia: a prospective observational study. Open Forum Infect Dis 2020;7 (12): ofaa563

42 Qin W, Dong F, Zhang Z, et al. Low molecular weight heparin and 28day mortality among patients with coronavirus disease 2019: a cohort study in the early epidemic era. Thromb Res 2020;198:19-22

43 Jonmarker S, Hollenberg J, Dahlberg M, et al. Dosing of thromboprophylaxis and mortality in critically ill COVID-19 patients. Crit Care 2020;24(01):653

44 Canoglu K, Saylan B. Therapeutic dosing of low-molecular-weight heparin may decrease mortality in patients with severe COVID-19 infection. Ann Saudi Med 2020;40(06):462-468

45 Rentsch CT, Beckman JA, Tomlinson L, Gellad WF, Alcorn C, Kidwai-Khan F. Early initiation of prophylactic anticoagulation for prevention of COVID-19 mortality: a nationwide cohort study of hospitalized patients in the United States. medRxiv. Doi: $10.1101 / 2020.12 .09 .20246579$

46 Martinelli I, Ciavarella A, Abbattista M, et al. Increasing dosages of low-molecular-weight heparin in hospitalized patients with Covid-19 [ePub ahead of print, 2021 Jan 3]. Intern Emerg Med 2021:1-7. Doi:10.1007/s11739-020-02585-9

47 Shen L, Qiu L, Liu D, et al. The association of low molecular weight heparin use and in-hospital mortality among patients hospitalized with COVID-19 [ePub ahead of print, 2021 Jan 4]. Cardiovasc Drugs Ther 2021:1-8. Doi:10.1007/s10557-02007133-3

48 Di Castelnuovo A, Costanzo S, Antinori A. Heparin in COVID-19 patients is associated with reduced in-hospital mortality: the multicentre Italian CORIST Study [ePub ahead of print, 2021]. Thromb Haemost 2021. Doi:10.1055/a-1347-6070

49 Wells GA, Shea B, O'Connell D, et al. The Newcastle-Ottawa Scale (NOS) for assessing the quality of nonrandomised studies in metaanalyses. Assessed February 4, 2021 at: http://www.ohri.ca/programs/clinical_epidemiology/oxford.asp

50 Viswanathan M, Ansari MT, Berkman ND, et al. Assessing the risk of bias of individual studies in systematic reviews of health care interventions. 2012 Mar 8. In: Methods Guide for Effectiveness and Comparative Effectiveness Reviews [Internet]. Rockville, MD: Agency for Healthcare Research and Quality (US); 2008-. PMID: 22479713

51 Lu YF, Pan LY, Zhang WW, et al. A meta-analysis of the incidence of venous thromboembolic events and impact of anticoagulation on mortality in patients with COVID-19. Int J Infect Dis 2020; 100:34-41

52 Salah HM, Naser JA, Calcaterra G, Bassareo PP, Mehta JL. The effect of anticoagulation use on mortality in COVID-19 infection. Am J Cardiol 2020;134:155-157

53 Kamel AM, Sobhy M, Magdy N, Sabry N, Farid S. Anticoagulation outcomes in hospitalized Covid-19 patients: a systematic review and meta-analysis of case-control and cohort studies. Rev Med Virol 2020;2180:e2180

54 Bousquet G, Falgarone G, Deutsch D, et al. ADL-dependency, Ddimers, LDH and absence of anticoagulation are independently associated with one-month mortality in older inpatients with Covid-19. Aging (Albany NY) 2020;12(12):11306-11313

55 Chen F, Sun W, Sun S, Li Z, Wang Z, Yu L. Clinical characteristics and risk factors for mortality among inpatients with COVID-19 in Wuhan, China. Clin Transl Med 2020;10(02):e40

56 Giacomelli A, Ridolfo AL, Milazzo L, et al. 30-day mortality in patients hospitalized with COVID-19 during the first wave of the Italian epidemic: a prospective cohort study. Pharmacol Res 2020; 158:104931

57 Khalil K, Agbontaen K, McNally D, et al. Clinical characteristics and 28-day mortality of medical patients admitted with COVID19 to a central London teaching hospital. J Infect 2020;81(03): e85-e89 